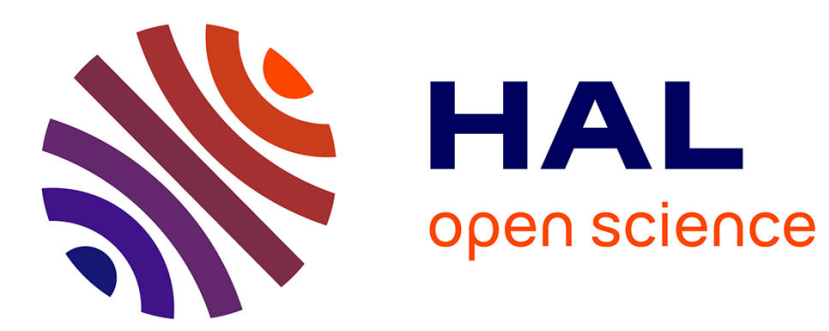

\title{
NULL CONTROLLABILITY OF THE STRUCTURALLY DAMPED WAVE EQUATION ON THE TWO-DIMENSIONAL TORUS
}

Patricio Guzmán, Lionel Rosier

\section{To cite this version:}

Patricio Guzmán, Lionel Rosier. NULL CONTROLLABILITY OF THE STRUCTURALLY DAMPED WAVE EQUATION ON THE TWO-DIMENSIONAL TORUS. 2019. hal-02159903

\section{HAL Id: hal-02159903 https://hal.science/hal-02159903}

Preprint submitted on 19 Jun 2019

HAL is a multi-disciplinary open access archive for the deposit and dissemination of scientific research documents, whether they are published or not. The documents may come from teaching and research institutions in France or abroad, or from public or private research centers.
L'archive ouverte pluridisciplinaire HAL, est destinée au dépôt et à la diffusion de documents scientifiques de niveau recherche, publiés ou non, émanant des établissements d'enseignement et de recherche français ou étrangers, des laboratoires publics ou privés. 


\title{
NULL CONTROLLABILITY OF THE STRUCTURALLY DAMPED WAVE EQUATION ON THE TWO-DIMENSIONAL TORUS
}

\author{
PATRICIO GUZMÁN AND LIONEL ROSIER
}

\begin{abstract}
We investigate the null controllability of the wave equation with a Kelvin-Voigt damping on the two-dimensional torus $\mathbb{T}^{2}$. We consider a distributed control supported in a moving domain $\omega(t)$ with a uniform motion at a constant velocity $c=(1, \zeta)$. The results we obtain depend strongly on the topological features of the geodesics of $\mathbb{T}^{2}$ with constant velocity $c$. When $\zeta \in \mathbb{Q}$, writing $\zeta=p / q$ with $p, q$ relatively prime, we prove that the null controllability holds if roughly the diameter of $\omega(0)$ is larger than $1 / p$ and if the control time is larger than $q$. We prove also that for almost every $\zeta \in \mathbb{R}_{+} \backslash \mathbb{Q}$, and also for some particular values including e.g. $\zeta=e$, the null controllability holds for any choice of $\omega(0)$ and for a sufficiently large control time. The proofs rely on a delicate construction of the weight function in a Carleman estimate which gets rid of a topogical assumption on the control region often encountered in the literature. Diophantine approximations are also needed when $\zeta$ is irrationnal.
\end{abstract}

2010 Mathematics Subject Classification: 35Q74, 93B05, 93B07, 93C20.

Keywords: Wave equation; Kelvin-Voigt damping; null controllability; parabolic-transport system; moving control; Carleman estimates; Diophantine approximations.

\section{INTRODUCTION}

We are here concerned with the null controllability of a classical model of viscoelasticity, namely the wave equation with both viscous Kelvin-Voigt damping and frictional damping. We consider a distributed control supported in a moving domain $\omega(t)$. The system reads

$$
\begin{aligned}
y_{t t}-\Delta y-v \Delta y_{t}+b(x) y_{t}=1_{\omega(t)}(x) u(x, t), & x \in \Omega, t \in(0, T), \\
y=0, & x \in \partial \Omega, t \in(0, T), \\
y(x, 0)=y_{0}(x), \quad y_{t}(x, 0)=y_{1}(x), & x \in \Omega .
\end{aligned}
$$

Here, $\Omega$ is a smooth, bounded, open set in $\mathbb{R}^{N}$ (where $N \geq 1$ ), $v>0$ is a viscous constant, $b \in L^{\infty}(\Omega$ ) is a function determining the frictional damping, and $u=u(x, t)$ stands for the control input. It is well known that system (1.1)-(1.3) fails to be null controllable when $\omega(t) \equiv \omega$ is a fixed open set in $\Omega$ with $\Omega \backslash \bar{\omega} \neq \emptyset$. This fact, noticed in [14] for $N=1$ (for boundary controls) is due to the existence of a limit point in the spectrum of the adjoint system. The same obstruction occurs for the Benjamin-Bona-Mahony (BBM) equation (see $[12,16])$ :

$$
y_{t}-y_{t x x}+y_{x}+y y_{x}=1_{\omega}(x) u(x, t) .
$$

To overcome this problem, L. Rosier and B.-Y. Zhang suggested in [16] to replace the fixed control region $\omega$ in (1.4) by a moving control region $\omega(t)$ that is allowed to visit the whole domain. More precisely, 
they proved that the system

$$
\begin{array}{cl}
y_{t}-y_{t x x}+y_{x}+y y_{x}=a(x-c t) u(x, t), & x \in \mathbb{T}, t \in(0, T), \\
y(x, 0)=y_{0}(x), & x \in \mathbb{T},
\end{array}
$$

where $\mathbb{T}=\mathbb{R} / \mathbb{Z}$ is the one-dimensional torus and $a \in C^{\infty}(\mathbb{T})$ is a nonnegative and not identically null function, is null controllable in time $T>1 /|c|$. Using the same kind of distributed control $a(x-c t) u(x, t)$, P. Martin, L. Rosier and P. Rouchon proved in [11] that the wave equation with structural damping

$$
y_{t t}-y_{x x}-y_{t x x}=a(x-c t) u(x, t), \quad x \in \mathbb{T},
$$

is null controllable in time $T>1 /|c|$.

The case of an open set $\Omega$ in $\mathbb{R}^{N}$ was considered by F. W. Chaves-Silva, L. Rosier and E. Zuazua in [4]. The null controllability of (1.1)-(1.3) was derived when $\omega(t)=X\left(\omega_{0}, t, 0\right)$, where $X$ is the flow generated by some vector field $f \in C\left([0, T], W^{2, \infty}\left(\mathbb{R}^{N}, \mathbb{R}^{N}\right)\right)$; that is, $X$ solves

$$
\left\{\begin{array}{l}
\frac{\partial X}{\partial t}\left(x, t, t_{0}\right)=f\left(X\left(x, t, t_{0}\right), t\right), \\
X\left(x, t_{0}, t_{0}\right)=x
\end{array}\right.
$$

For instance, with the choice $f(x, t)=\dot{\gamma}(t)$ for some $\gamma \in C^{1}\left([0, T], \mathbb{R}^{N}\right)$, we obtain

$$
X\left(x, t, t_{0}\right)=x+\gamma(t)-\gamma\left(t_{0}\right) .
$$

Actually, the results in [4] (and also those in [5]) were derived under the assumption that there exist a bounded, smooth, open set $\omega_{0} \subset \mathbb{R}^{N}$, a curve $\Gamma \in C^{\infty}\left([0, T], \mathbb{R}^{N}\right)$, and two times $t_{1}, t_{2}$ with $0 \leq t_{1} \leq t_{2} \leq T$ such that

$$
\begin{aligned}
& \Gamma(t) \in X\left(\omega_{0}, t, 0\right) \cap \Omega \quad \forall t \in[0, T] ; \\
& \bar{\Omega} \subset \bigcup_{t \in[0, T]} X\left(\omega_{0}, t, 0\right)=\left\{X(x, t, 0) ; x \in \omega_{0}, t \in[0, T]\right\} ; \\
& \Omega \backslash \overline{X\left(\omega_{0}, t, 0\right)} \text { is nonempty and connected for } t \in\left[0, t_{1}\right] \cup\left[t_{2}, T\right] ; \\
& \Omega \backslash \overline{X\left(\omega_{0}, t, 0\right)} \text { has two (nonempty) connected components for } t \in\left(t_{1}, t_{2}\right) ; \\
& \forall \gamma \in C([0, T], \Omega), \exists t \in[0, T], \gamma(t) \in X\left(\omega_{0}, t, 0\right) .
\end{aligned}
$$

More recently, F. W. Chavez Silva , X. Zhang and E. Zuazua investigated in [5] the null controllability of a heat equation with memory by using a moving control, namely the system

$$
\begin{aligned}
y_{t}-\Delta y+\int_{0}^{t} M(t-s) y(s) d s=1_{\omega(t)}(x) u(x, t), & x \in \Omega, t \in(0, T), \\
y=0, & x \in \partial \Omega, t \in(0, T), \\
y(x, 0)=0, & x \in \Omega .
\end{aligned}
$$

Assuming that (1.8)-(1.12) hold, that $M \in L^{1}(0, T)$ and picking an open set $\omega$ with $\overline{\omega_{0}} \subset \omega$, they proved that system (1.13)-(1.15) is null controllable in time $T$ by taking $\omega(t):=X(\omega, t, 0)$.

It would be desirable to obtain the null controllability of (1.1)-(1.3) assuming only (1.9), but such a result (if true) is still not available in the literature. It is quite obvious that the conditions (1.10) and (1.11) are not consequences of (1.9). On the other hand, it was noticed in [4] that (1.8) and (1.12) are not implied by (1.9). The most conservative condition seems to be (1.11), for the natural situation when 
a small ball $\omega(t)=B(\gamma(t), \varepsilon)$ visits the whole domain $\Omega$ (with $\varepsilon \ll 1$ so that (1.11) fails) is not covered by the theory developed in $[4,5]$.

The aim of the paper is to obtain almost sharp results, as far as the control region is concerned, for the null controllability of the wave equation with both Kelvin-Voigt and frictional damping. We shall focus on the case of the simple domain $\Omega=(0,1)^{N}$ with periodic boundary conditions (i.e. we shall assume that $x \in \mathbb{T}^{N}=\mathbb{R}^{N} / \mathbb{Z}^{N}$ ), and we take for the flow $X$ a uniform translation corresponding to $f(x, t)=c$ $\left(c \in \mathbb{R}^{N}\right.$ being a constant vector), i.e.

$$
X\left(x, t, t_{0}\right)=x+\left(t-t_{0}\right) c .
$$

Even if our results could be stated in any dimension $N \geq 1$, we shall restrict ourselves to the dimension $N=2$, for the sake of simplicity. We may, without loss of generality, assume that the viscous constant is $v=1$.

We are thus concerned with the null controllability of the system

$$
\begin{array}{cl}
y_{t t}-\Delta y-\Delta y_{t}+b(x) y_{t}=1_{\omega(t)}(x) u(x, t), & x \in \mathbb{T}^{2}, t \in(0, T), \\
y(x, .)=y_{0}(x), y_{t}(x, 0)=y_{1}(x), & x \in \mathbb{T}^{2} .
\end{array}
$$

We shall assume that

$$
\omega(t)=\omega+t c, \quad t \in \mathbb{R},
$$

where $c=\left(c_{1}, c_{2}\right) \neq(0,0)$ is a constant velocity and $\omega \subset \mathbb{T}^{2}$ is any open set with $\overline{\omega_{0}} \subset \omega$, $\omega_{0}$ being a nonempty open set. Without loss of generality, we can assume that

$$
\begin{aligned}
& (0,0) \in \omega_{0}, \\
& c=(1, \zeta),
\end{aligned}
$$

where $\zeta \in \mathbb{R}_{+}$. We shall assume throughout that

$$
\exists T>0, \quad \mathbb{T}^{2}=\bigcup_{t \in[0, T]} \omega(t) .
$$

We shall denote by $\pi$ the projection from $\mathbb{R}^{2}$ onto $\mathbb{T}^{2}$, defined as $\pi(x)=x+\mathbb{Z}^{2}$ for all $x \in \mathbb{R}^{2}$.

Our aim is the derivation of null controllability results for (1.16)-(1.17) with $\omega(t)$ as in (1.18), that are sharp as far as the geometry of $\omega_{0}$ is concerned. We shall investigate the three possible cases: (i) $c=(1,0)$; (ii) $c=(1, \zeta)$ with $\zeta \in \mathbb{Q}_{+}^{*}$; (ii) $c=(1, \zeta)$ with $\zeta \in \mathbb{R}_{+} \backslash \mathbb{Q}$.

Let us state the main results in this paper.

(i) Assume that $c=(1,0)$. Note that the condition (1.21) yields

$$
\left\{x_{2} \in \mathbb{T} ; \exists x_{1} \in \mathbb{T},\left(x_{1}, x_{2}\right) \in \omega_{0}\right\}=\mathbb{T} .
$$

In other words, the projection along the $x_{2}$-axis of the control region at $t=0$ should be the whole domain $\mathbb{T}$. We shall see that, for an open set $\omega_{0}$ which is roughly delimited by two curves $x_{1}=\rho_{i}\left(x_{2}\right), i=1,2$, this condition is also sufficient. (See Figure 1.)

The first result is this paper is a null controllability result for (1.16)-(1.17) when $c=(1,0)$.

Theorem 1.1. Assume that $c=(1,0)$. Assume given two functions $\rho_{1}, \rho_{2} \in C([0,1], \mathbb{R})$ such that $\rho_{i}(0)=$ $\rho_{i}(1)$ for $i=1,2$, and $0<\rho_{1}\left(x_{2}\right)<\rho_{2}\left(x_{2}\right)<1$ for $x_{2} \in[0,1]$, and let

$$
\omega_{0}=\pi\left\{x=\left(x_{1}, x_{2}\right) \in[0,1]^{2} ; \rho_{1}\left(x_{2}\right)<x_{1}<\rho_{2}\left(x_{2}\right)\right\} .
$$




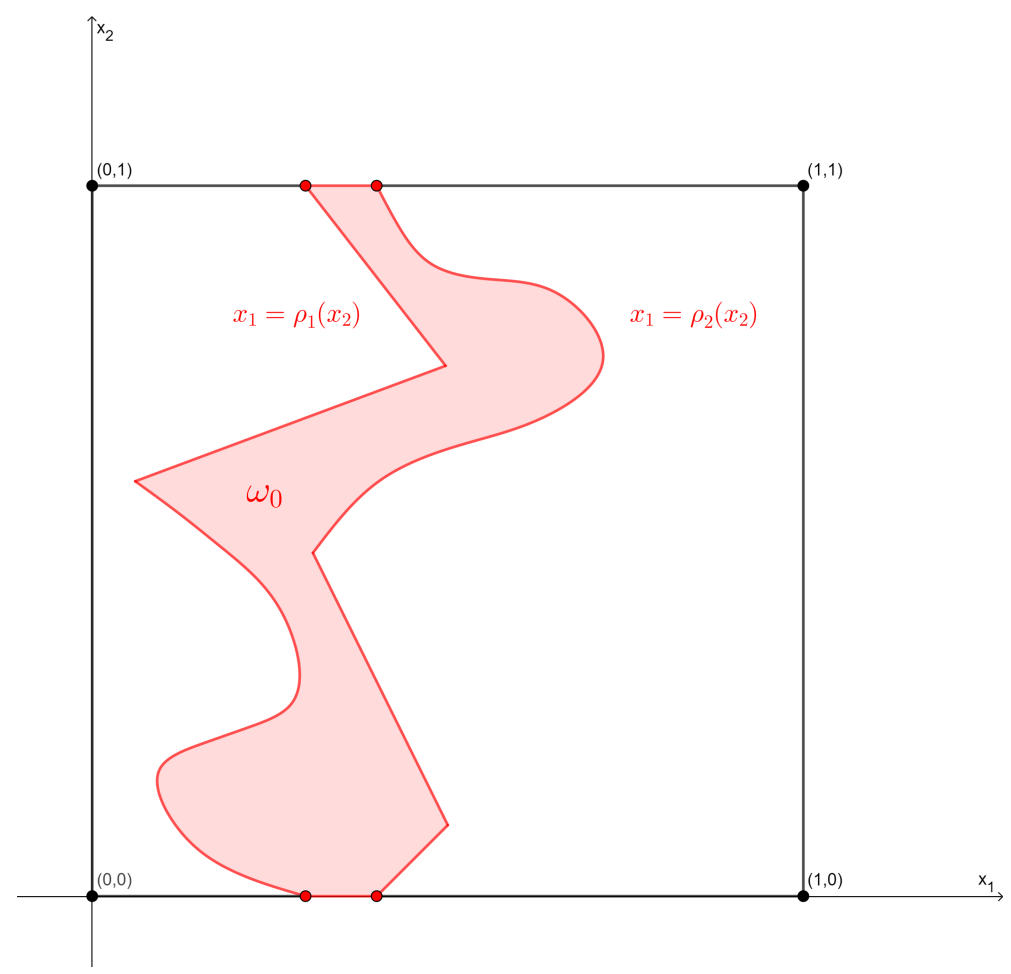

FIGURE 1. The shape of $\omega_{0}$ when $c=(1,0)$.

Pick any time $T>1-\min _{x_{2} \in[0,1]} \rho_{2}\left(x_{2}\right)+\max _{x_{2} \in[0,1]} \rho_{1}\left(x_{2}\right)$ Let $\omega$ be any open set in $\mathbb{T}^{2}$ such that $\overline{\omega_{0}} \subset \omega$, and let $(\omega(t))_{t \in[0, T]}$ be as in (1.18). Then for any $\left(y_{0}, y_{1}\right) \in H^{2}\left(\mathbb{T}^{2}\right) \times L^{2}\left(\mathbb{T}^{2}\right)$ there exists a control $u \in L^{2}\left(0, T, L^{2}\left(\mathbb{T}^{2}\right)\right)$ such that the solution y of (1.16)-(1.17) satisfies $y(., T)=y_{t}(., T)=0$.

Note that the control time is sharp when the functions $\rho_{1}$ and $\rho_{2}$ assume constant values, for we get the condition $T>1-\left(\rho_{2}-\rho_{1}\right)$.

(ii) Assume now that $c=\left(1, \frac{p}{q}\right)$ with

$$
p, q \in \mathbb{N}^{*}, p \text { and } q \text { being relatively prime. }
$$

Then it is well known that the curve $x \rightarrow \pi(c t)$ is $q$-periodic (hence closed), and that its image $\pi\{c t ; t \in$ $0, q]\}$ is homeomorphic to a 1 -sphere (i.e. a circle). It is compact and not dense in $\mathbb{T}^{2}$. (See Figure 2.)

It is expected that the extension of $\omega_{0}$ along the transversal variable $x_{1}$ should be sufficiently large for (1.16)-(1.17) to be null controllable. A precise statement of this claim is given in the following proposition.

Proposition 1.1. Assume that $c=\left(1, \frac{p}{q}\right)$ with $p$ and $q$ as in (1.22). Let $L$ and $\varepsilon$ be positive numbers such that the set

$$
\omega_{0}=\pi([0, L] \times\{0\}+B((0,0), \varepsilon))
$$




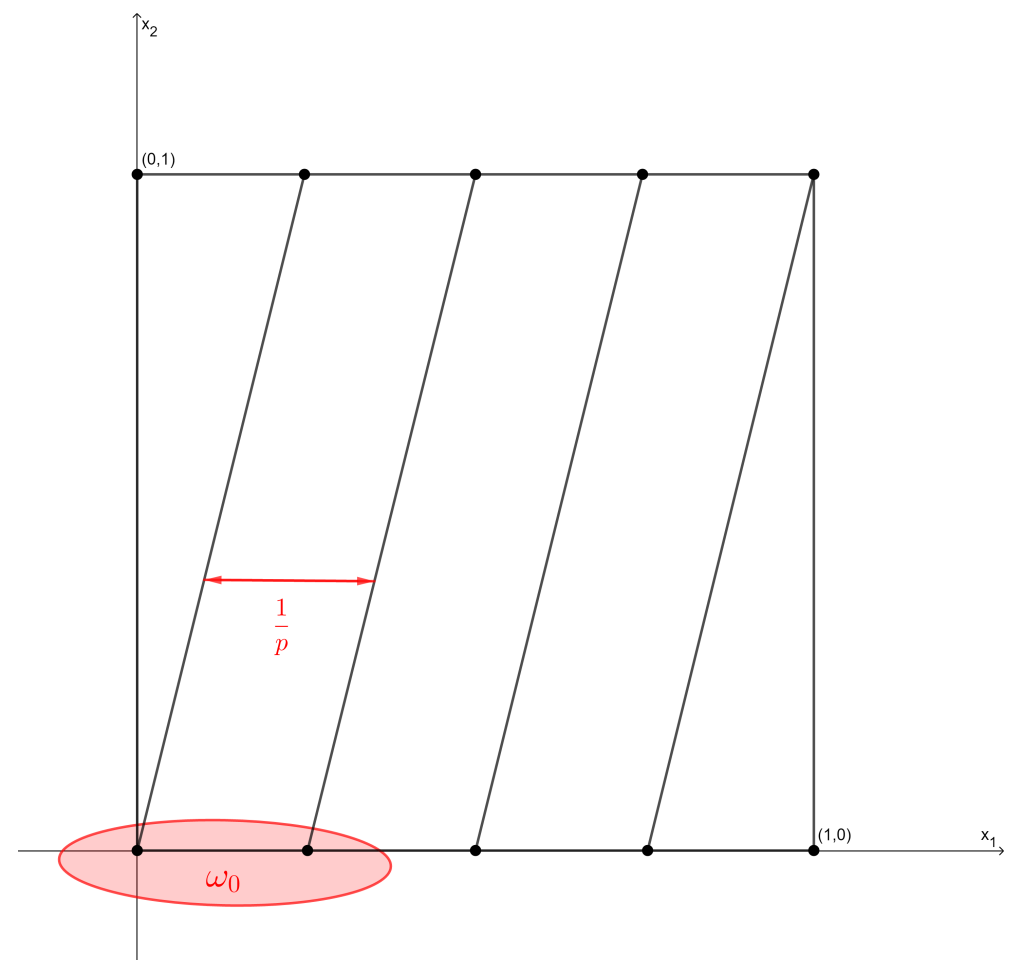

FIGURE 2. The shape of $\omega_{0}$ when $c=(1, p / q)$ for $(p, q)=(4,1)$.

satisfies (1.21) with $\omega(t)$ given by (1.18). Then

$$
L \geq \frac{1-2 \sqrt{p^{2}+q^{2}} \varepsilon-\pi \varepsilon^{2}}{p+2 \varepsilon} .
$$

In particular, $L \geq \frac{1}{p}+O(\varepsilon)$ as $\varepsilon \rightarrow 0^{+}$.

Thus, the extension of $\omega_{0}$ along the $x_{1}$-variable should be at least of order $1 / p$. Conversely, we shall prove that under a similar condition the null controllability of (1.16)-(1.17) in large time can be derived. This is the content of the following theorem, which is the first main result in this paper.

Theorem 1.2. Assume that $c=\left(1, \frac{p}{q}\right)$ where $p$ and $q$ are as in (1.22). Assume that $\omega_{0} \subset \mathbb{T}^{2}$ is an open set with

$$
\pi\left(\left[0, \frac{1}{p}\right] \times\{0\}\right) \subset \omega_{0} .
$$

Let $\omega$ be any open set in $\mathbb{T}^{2}$ such that $\overline{\omega_{0}} \subset \omega$, and let $(\omega(t))_{t \in[0, T]}$ be as in (1.18). Pick any $T>q$. Then for any $\left(y_{0}, y_{1}\right) \in H^{2}\left(\mathbb{T}^{2}\right) \times L^{2}\left(\mathbb{T}^{2}\right)$, there exists a control $u \in L^{2}\left(0, T, L^{2}\left(\mathbb{T}^{2}\right)\right)$ such that the solution $y$ of (1.16)-(1.17) satisfies $y(., T)=y_{t}(., T)=0$.

Remark 1.1. (1) Theorem 1.2 is sharp as far as both the control domain and the time control are concerned. For the control domain, this follows from Proposition 1.1, and for the time control, 
this follows from the observation that

$$
\pi\left\{s\left(\frac{1}{p}, 1\right)+t\left(1, \frac{p}{q}\right) ; s \in[0,1], t \in[0, T]\right\}=\mathbb{T}^{2} \Longleftrightarrow T \geq q .
$$

(2) Theorem 1.2 applies when $\omega_{0}=B(0, r)$ for $r>(2 p)^{-1}$ (the position of the center of the ball being irrelevant).

(iii) Assume that $c=(1, \zeta)$ with $\zeta \in \mathbb{R}_{+} \backslash \mathbb{Q}$. Then it is well known that the curve $t \rightarrow \pi(t c)$ is one-to-one on $\mathbb{R}$, and that its image $\{\pi(t c) ; t \in \mathbb{R}\}$ is dense in $\mathbb{T}^{2}$. It is thus natural to expect that for any nonempty open set $\omega_{0} \subset \mathbb{T}^{2}$, the set

$$
\omega_{0}+[0, T] c=\left\{x+t c ; x \in \omega_{0}, t \in[0, T]\right\}
$$

covers $\mathbb{T}^{2}$ if the control time $T>0$ is large enough. (See Figure 3.)

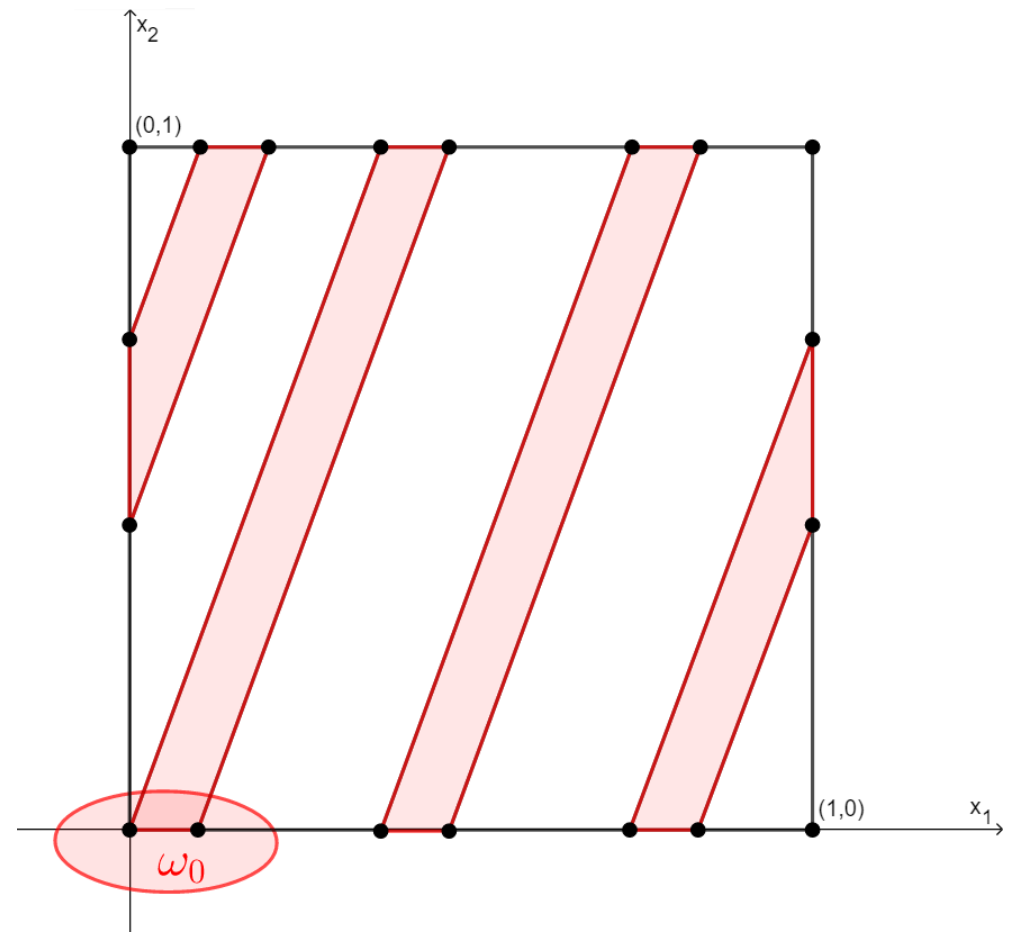

FIGURE 3. The case $c=(1, \zeta)$ with $\zeta \in \mathbb{R}_{+} \backslash \mathbb{Q}$.

The following proposition shows that it is indeed the case.

Proposition 1.2. If $c=(1, \zeta)$ with $\zeta \in \mathbb{R}_{+} \backslash \mathbb{Q}$, then for any nonempty open set $\omega_{0} \subset \mathbb{T}^{2}$, there exists some $T>0$ such that

$$
\omega_{0}+[0, T] c=\mathbb{T}^{2} .
$$

The above proposition suggests that the control system (1.16)-(1.17) may be null controllable for any control region but in large time. Such a result can be established for almost every $\zeta \in \mathbb{R}_{+} \backslash \mathbb{Q}$. To state it, we need to introduce a class of irrational numbers that can be approximated by rational numbers with an error less than $\delta / q^{2}$. 
Definition 1.1. For any $\delta>0$, let $\mathrm{J}_{\delta}$ denote the set of numbers $\zeta \in \mathbb{R}_{+} \backslash \mathbb{Q}$ such that for all $A>0$, there exists a pair $(p, q)$ as in (1.22) with $q \geq A$ and

$$
\left|\zeta-\frac{p}{q}\right| \leq \frac{\delta}{q^{2}}
$$

It is well known that $\mathcal{J}_{1 / \sqrt{5}}=\mathbb{R}_{+} \backslash \mathbb{Q}$, while $(\sqrt{5}-1) / 2 \notin \mathcal{J}_{\delta}$ for $\delta<1 / \sqrt{5}$ (see e.g. [9, Theorem 193 and Theorem 194]). Furthermore, it is known that $\left(\mathbb{R}_{+} \backslash \mathbb{Q}\right) \backslash \mathcal{J}_{\delta}$ is of measure zero for any $\delta>0$ (see e.g. [9, Theorem 196]).

The following result is the second main result in this paper.

Theorem 1.3. Assume that $c=(1, \zeta)$ where $\zeta \in \mathcal{J}_{\delta}$ for some $\delta>0$ with

$$
f_{2}(\delta, \zeta):=\left(32+\frac{8}{\zeta}\right) \delta+\left(549+\frac{64}{\zeta}+\frac{8}{\zeta^{2}}\right) \delta^{2}<1 .
$$

Let $\omega$ be any nonempty open set in $\mathbb{T}^{2}$, and let $(\omega(t))_{t \in[0, T]}$ be as in (1.18). Then there exists a time $T>0$ such that for any $\left(y_{0}, y_{1}\right) \in H^{2}\left(\mathbb{T}^{2}\right) \times L^{2}\left(\mathbb{T}^{2}\right)$, there exists a control $u \in L^{2}\left(0, T, L^{2}\left(\mathbb{T}^{2}\right)\right)$ such that the solution $y$ of (1.16)-(1.17) satisfies $y(., T)=y_{t}(., T)=0$.

Remark 1.2. (1) Theorem 1.3 applies for a.e. $\zeta \in \mathbb{R}_{+} \backslash \mathbb{Q}$, for the set $\left(\mathbb{R}_{+} \backslash \mathbb{Q}\right) \backslash \cap_{n \geq 1} \mathcal{J}_{1 / n}$ is of measure zero.

(2) Theorem 1.3 applies also when $\zeta \in \mathcal{J}_{\delta}$ with $\zeta \geq 1$ and $\delta \leq 10^{-2}$. Indeed, $f_{2}(\delta, \zeta) \leq f_{2}(\delta, 1)=$ $40 \delta+621 \delta^{2}$. The issue whether Theorem 1.3 is actually true for any $\zeta \in \mathbb{R}_{+} \backslash \mathbb{Q}$ is open and challenging.

(3) We can provide explicit examples of numbers to which Theorem 1.3 applies.

For a given $\zeta \in \mathbb{R}_{+} \backslash \mathbb{Q}$, we introduce its continued fraction (see e.g. [9]) $\left[a_{0}, a_{1}, a_{2}, \ldots\right]$ where $a_{0} \in \mathbb{N}, a_{i} \in \mathbb{N}^{*}$ for $i \geq 1$ and $\zeta=\lim _{n \rightarrow+\infty}\left[a_{0}, a_{1}, \ldots, a_{n}\right]$ with

$$
\left[a_{0}, a_{1}, \ldots, a_{n}\right]=a_{0}+\frac{1}{a_{1}+\frac{1}{a_{2}+\frac{1}{\cdots+\frac{1}{a_{n}}}}} .
$$

Denoting $\left[a_{0}, a_{1}, \ldots, a_{n}\right]=p_{n} / q_{n}$ with $p_{n}$ and $q_{n}$ as in (1.22), then $q_{n} \rightarrow \infty$ and

$$
\left|\zeta-\frac{p_{n}}{q_{n}}\right| \leq \frac{1}{a_{n+1} q_{n}^{2}}
$$

(See e.g. [9, Section 10.9].) Thus $\xi \in \mathcal{J}_{\delta}$ if $a_{n}>\delta^{-1}$ for infinitely many $n$. Let us give some examples of numbers $\zeta$ to which Theorem 1.3 applies. The first one is an algebraic number, the second one and the third one are transcendental numbers.

(a) Let $\zeta=\sqrt{m^{2}+1}$ with $m \in \mathbb{N}$. Then $\zeta=[m, 2 m, 2 m, 2 m, \ldots]$ so that $\zeta \in \mathcal{J}_{\delta}$ if $2 m>\delta^{-1}$, and hence Theorem 1.3 applies for $m>50$.

(b) Let $\zeta=L=\sum_{k=1}^{\infty} 10^{-k !}$ (Liouville's constant). Then $L \in \mathcal{J}_{\delta}$ for all $\delta>0$. Indeed, setting $q_{n}=10^{n !}$ and $p_{n}=\sum_{k=1}^{n} 10^{n !-k !}$ for $n \in \mathbb{N}^{*}$, for given $A, \delta>0$ we notice that $\left(p_{n}, q_{n}\right)$ is (for $n$ large enough) as in (1.22) with $q_{n}>A$ and

$$
\left|L-\frac{p_{n}}{q_{n}}\right|=\sum_{k=n+1}^{\infty} 10^{-k !} \leq \frac{2}{10^{(n+1) !}} \leq \frac{\delta}{q_{n}^{2}} .
$$


(c) Let $\zeta=e=\exp (1)$. Then Euler proved that $e=[2,1,2,1,1,4,1,1,6,1,1,8, \ldots]$ (see e.g. [10]). Thus $a_{3 n-1}=2 n$ for all $n \in \mathbb{N}^{*}$, so that $e \in \mathcal{J}_{\delta}$ for all $\delta>0$.

Let us say a few words about the proofs of Theorems 1.1, 1.2 and 1.3. Following [4], we decompose (1.16)-(1.17) into a system coupling a parabolic equation with an ordinary differential equation (ODE), namely

$$
\begin{aligned}
y_{t}-\Delta y+(b(x)-1) y=z, & (t, x) \in(0, T) \times \mathbb{T}^{2}, \\
z_{t}+z=1_{\omega(t)}(x) u+(b(x)-1) y, & (t, x) \in(0, T) \times \mathbb{T}^{2}, \\
y(x, 0)=y_{0}(x), & x \in \mathbb{T}^{2}, \\
z(x, 0)=y_{1}(x)-\Delta y_{0}(x)+(b(x)-1) y_{0}(x), & x \in \mathbb{T}^{2} .
\end{aligned}
$$

Introducing the new unknown functions

$$
\begin{aligned}
& h(x, t):=y(x+t c, t), \\
& k(x, t):=z(x+t c, t),
\end{aligned}
$$

we see that system (1.26)-(1.29) is transformed into the system:

$$
\begin{aligned}
h_{t}-\Delta h-c \cdot \nabla h+(b(x+t c)-1) h=k, & (t, x) \in(0, T) \times \mathbb{T}^{2}, \\
k_{t}-c \cdot \nabla k+k=1_{\omega_{0}}(x) u(x+t c, t)+(b(x+t c)-1) h, & (t, x) \in(0, T) \times \mathbb{T}^{2}, \\
h(x, 0)=y_{0}(x), & x \in \mathbb{T}^{2}, \\
k(x, 0)=y_{1}(x)-\Delta y_{0}(x)+(b(x)-1) y_{0}(x), & x \in \mathbb{T}^{2} .
\end{aligned}
$$

for which the control input is supported in the fixed domain $\omega_{0}$.

Next, the corresponding adjoint system to (1.30)-(1.33) reads

$$
\begin{aligned}
-v_{t}-\Delta v+c \cdot \nabla v+(b(x+t c)-1) v=(b(x+t c)-1) w, & (t, x) \in(0, T) \times \mathbb{T}^{2}, \\
-w_{t}+c \cdot \nabla w+w=v, & (t, x) \in(0, T) \times \mathbb{T}^{2}, \\
v(x, T)=v_{T}(x), & x \in \mathbb{T}^{2}, \\
w(x, T)=w_{T}(x), & x \in \mathbb{T}^{2} .
\end{aligned}
$$

By classical duality arguments, the null controllability of (1.30)-(1.33) is proved whenever we have established the following observability inequality for the adjoint system (1.34)-(1.37):

$$
\int_{\mathbb{T}^{2}}\left(|v(x, 0)|^{2}+|w(x, 0)|^{2}\right) d x \leq C_{o b s} \int_{0}^{T} \int_{\omega_{0}}|w(x, t)|^{2} d x d t,
$$

for some constant $C_{o b s}>0$ and all $v_{T}, w_{T} \in L^{2}\left(\mathbb{T}^{2}\right)$. In order to establish the observability inequality (1.38), following [1,4], we derive Carleman estimates for the backward heat equation (1.34) and for the transport equation (1.35) with the same weights functions. To get "almost sharp" results for the geometry of the control region, the construction of the weight functions in the Carleman estimates turns out to be much more delicate than in [4]. To derive Theorem 1.2, we need to prove the existence of a function $\psi_{0} \in C^{\infty}\left(\mathbb{T}^{2}\right)$ such that

$$
\begin{aligned}
\nabla \psi_{0}(x) \neq 0, & \forall x \in \mathbb{T}^{2} \backslash \omega_{0}, \\
c \cdot \nabla\left(c \cdot \nabla \psi_{0}\right)(x)>0, & \forall x \in \mathbb{T}^{2} \backslash \omega_{0} .
\end{aligned}
$$


This is done by viewing $\psi_{0}$ as a smooth $\mathbb{Z}^{2}$-periodic function which is constructed explicitly on the domain

$$
D:=\left\{s\left(\frac{1}{p}, 0\right)+t\left(1, \frac{p}{q}\right), s \in[0,1), t \in[0, q)\right\},
$$

and by noticing that $D$ is a fundamental domain for the action of the group $\left(\mathbb{Z}^{2},+\right)$; that is to say, the family $(D+(m, n))_{(m, n) \in \mathbb{Z}^{2}}$ constitutes a partition of $\mathbb{R}^{2}$.

The proof of Theorem 1.3 cannot be done along the same lines as for Theorem 1.2, for no fundamental domain similar to $D$ is available. However, approximating $\zeta \in \mathbb{R}_{+} \backslash \mathbb{Q}$ by some rational number $p / q$ as in (1.25) and using Theorem 1.2, we can construct a function $\psi_{0}$ satisfying (1.39)-(1.40).

A few words should be said about what we mean by a solution of system (1.16)-(1.17) or, equivalently, by a solution of system (1.26)-(1.29). The following result follows from the classical semigroup theory (see e.g. [13]). Its proof is omitted.

Proposition 1.3. The operator

$$
A(y, z)=(\Delta y-(b(x)-1) y+z,-z+(b(x)-1) y),
$$

with domain $D(A)=H^{2}\left(\mathbb{T}^{2}\right) \times L^{2}\left(\mathbb{T}^{2}\right) \subset\left[L^{2}\left(\mathbb{T}^{2}\right)\right]^{2}$ generates a strongly semigroup $\left(e^{t A}\right)_{t \geq 0}$ in $\left[L^{2}\left(\mathbb{T}^{2}\right)\right]^{2}$. As a consequence, for any $\left(y_{0}, z_{0}\right) \in\left[L^{2}\left(\mathbb{T}^{2}\right)\right]^{2}$ and any $u \in L^{2}\left(0, T, L^{2}\left(\mathbb{T}^{2}\right)\right)$, setting $F(x, t)=\left(0,1_{\omega(t)}(x)\right.$ $u(x, t))$, we have that the system

$$
(y, z)_{t}=A(y, z)+F, \quad(y, z)(., 0)=\left(y_{0}, z_{0}\right),
$$

admits a unique mild solution $(y, z) \in C\left([0, T],\left[L^{2}\left(\mathbb{T}^{2}\right)\right]^{2}\right)$. Furthermore, $y \in L^{2}\left(0, T ; H^{1}\left(\mathbb{T}^{2}\right)\right)$.

Going back to system (1.16)-(1.17) and using (1.26), we see that for any $\left(y_{0}, y_{1}\right) \in H^{2}\left(\mathbb{T}^{2}\right) \times L^{2}\left(\mathbb{T}^{2}\right)$ and any control input $u \in L^{2}\left(0, T, L^{2}\left(\mathbb{T}^{2}\right)\right)$, we can construct a solution $y \in C\left([0, T], L^{2}\left(\mathbb{T}^{2}\right)\right)$ of (1.16)(1.17) with $y_{t}-\Delta y \in C\left([0, T], L^{2}\left(\mathbb{T}^{2}\right)\right)$.

The paper is organized as follows. Section 2 is devoted to the construction of a function $\psi_{0}$ as in (1.39)-(1.40) in each of the cases (i), (ii), and (iii). In Section 3, we state and prove some Carleman estimates for a transport equation and for a backward heat equation with the same weights involving the function $\psi_{0}$ in their expressions. In Section 4, we derive the observability inequality for the adjoint system (1.34)-(1.37) and next complete the proofs of Theorems 1.1, 1.2, and 1.3.

\section{CONSTRUCTION OF THE FUNCTION $\psi_{0}$}

In this section, we prove the existence of a function $\psi_{0} \in C^{\infty}\left(\mathbb{T}^{2}\right)$ satisfying (1.39)-(1.40) for $c$ and $\omega_{0}$ as in Theorems 1.1, 1.2, and 1.3, respectively. Let us consider successively the cases (i) $c=(1,0)$; (ii) $c=(1, p / q)\left(p, q \in \mathbb{N}^{*}\right)$; and (iii) $c=(1, \zeta)\left(\zeta \in \mathbb{R}_{+} \backslash \mathbb{Q}\right)$.

(i) $c=(1,0)$

Proposition 2.1. Let $c$ and $\omega_{0}$ be as in Theorem 1.1, and let $\tau>1-\min _{x_{2} \in[0,1]} \rho_{2}\left(x_{2}\right)>0$. Then there exists a nonnegative function $\psi_{0} \in C^{\infty}\left(\mathbb{T}^{2}\right)$ satisfying

$$
\begin{array}{r}
2\left(\tau-1+\min _{x_{2} \in[0,1]} \rho_{2}\left(x_{2}\right)\right) \leq c \cdot \nabla \psi_{0} \leq 2\left(\tau+\max _{x_{2} \in[0,1]} \rho_{1}\left(x_{2}\right)\right), \quad \text { in } \mathbb{T}^{2} \backslash \omega_{0}, \\
c \cdot \nabla\left(c \cdot \nabla \psi_{0}\right)=2, \quad \text { in } \mathbb{T}^{2} \backslash \omega_{0} .
\end{array}
$$


Proof. Let $\rho_{1}$ and $\rho_{2}$ be as in Theorem 1.1. Recall that $\rho_{1}(0)=\rho_{1}(1)$ and $\rho_{2}(0)=\rho_{2}(1)$. Introduce the open sets in $\mathbb{T}^{2}$ :

$$
\begin{aligned}
& \Omega_{1}:=\left\{\pi(x) ; x=\left(x_{1}, x_{2}\right) \in[0,1]^{2}, \frac{2}{3} \rho_{1}\left(x_{2}\right)+\frac{1}{3} \rho_{2}\left(x_{2}\right)<x_{1}<\frac{1}{3} \rho_{1}\left(x_{2}\right)+\frac{2}{3} \rho_{2}\left(x_{2}\right)\right\}, \\
& \Omega_{2}:=\left\{\pi(x) ; x=\left(x_{1}, x_{2}\right) \in[0,1]^{2}, \frac{4}{5} \rho_{1}\left(x_{2}\right)+\frac{1}{5} \rho_{2}\left(x_{2}\right)<x_{1}<\frac{1}{5} \rho_{1}\left(x_{2}\right)+\frac{4}{5} \rho_{2}\left(x_{2}\right)\right\} .
\end{aligned}
$$

Since $\overline{\Omega_{1}} \subset \Omega_{2}$, we may pick (see e.g. [8, Corollary 4.7]) a function $\chi \in C^{\infty}\left(\mathbb{T}^{2}\right)$ such that $0 \leq \chi \leq 1$ and

$$
\chi(x)= \begin{cases}0 & \text { if } x \in \Omega_{1}, \\ 1 & \text { if } x \notin \Omega_{2}\end{cases}
$$

For $x=\left(x_{1}, x_{2}\right) \in[0,1]^{2}$, we set

$$
\psi_{0}(x)= \begin{cases}\chi(\pi(x))\left(x_{1}+\tau\right)^{2} & \text { if } x_{1} \leq \frac{1}{2}\left(\rho_{1}\left(x_{2}\right)+\rho_{2}\left(x_{2}\right)\right), \\ \chi(\pi(x))\left(x_{1}-1+\tau\right)^{2} & \text { if } x_{1}>\frac{1}{2}\left(\rho_{1}\left(x_{2}\right)+\rho_{2}\left(x_{2}\right)\right) .\end{cases}
$$

Then it is clear that $\psi_{0}$ can be extended as a $\mathbb{Z}^{2}$-periodic smooth function (i.e. $\psi_{0} \in C^{\infty}\left(\mathbb{T}^{2}\right)$ ). On the other hand,

and hence

$$
c \cdot \nabla \psi_{0}(x)=\frac{\partial \psi_{0}}{\partial x_{1}}= \begin{cases}2\left(x_{1}+\tau\right) & \text { if } 0 \leq x_{1} \leq \rho_{1}\left(x_{2}\right), \\ 2\left(x_{1}-1+\tau\right) & \text { if } \rho_{2}\left(x_{2}\right) \leq x_{1} \leq 1,\end{cases}
$$

$$
\begin{aligned}
2 \tau \leq c \cdot \nabla \psi_{0}(x) \leq 2\left(\tau+\max _{x_{2} \in[0,1]} \rho_{1}\left(x_{2}\right)\right), & \text { if } 0 \leq x_{1} \leq \rho_{1}\left(x_{2}\right), \\
2\left(\tau-1+\min _{x_{2} \in[0,1]} \rho_{2}\left(x_{2}\right)\right) \leq c \cdot \nabla \psi_{0}(x) \leq 2 \tau, & \text { if } \rho_{2}\left(x_{2}\right) \leq x_{1} \leq 1,
\end{aligned}
$$

so that (2.1) holds. Finally,

$$
c \cdot \nabla\left(c \cdot \nabla \psi_{0}\right)=\frac{\partial^{2} \psi_{0}}{\partial x_{1}^{2}}=2 \quad \text { if } \quad x_{1} \notin\left(\rho_{1}\left(x_{2}\right), \rho_{2}\left(x_{2}\right)\right),
$$

so that (2.2) holds as well.

(ii) $c=\left(1, \frac{p}{q}\right), p, q \in \mathbb{N}^{*}, p$ and $q$ relatively prime.

Before performing the construction of the function $\psi_{0}$, we first prove Proposition 1.1.

Proof of Proposition 1.1: From (1.21) and the fact that

$$
\pi\left(q\left(1, \frac{p}{q}\right)\right)=\pi(q, p)=\pi(0,0) \in \omega_{0},
$$

we infer that

Introduce the set

$$
\omega_{0}+[0, q]\left(1, \frac{p}{q}\right)=\mathbb{T}^{2}
$$

and its $\varepsilon$-neighborhood

$$
K:=\left\{s(L, 0)+t\left(1, \frac{p}{q}\right) ; s \in[0,1], t \in[0, q]\right\} \subset \mathbb{R}^{2}
$$

$$
K_{\varepsilon}:=\left\{(x, y) \in \mathbb{R}^{2} ; \operatorname{dist}((x, y), K)<\varepsilon\right\}=K+B((0,0), \varepsilon) .
$$


Then it follows from the definitions of $\omega_{0}$ and $K_{\varepsilon}$ that

$$
\mathbb{T}^{2}=\omega_{0}+[0, q]\left(1, \frac{p}{q}\right)=\pi\left(K_{\varepsilon}\right) .
$$

This yields $1=\lambda\left([0,1]^{2}\right) \leq \lambda\left(K_{\varepsilon}\right)$, where $\lambda$ denotes Lebesgue measure. Elementary computations give

$$
\lambda\left(K_{\varepsilon}\right)=L p+2 \varepsilon L+2 \varepsilon \sqrt{p^{2}+q^{2}}+\pi \varepsilon^{2},
$$

and (1.23) follows.

Introduce some notations. For any real number $R$, let $[R]$ (resp. $\{R\}$ ) denote its integral part (resp. its fractional part); that is, $[R]:=\sup \{n \in \mathbb{Z} ; n \leq R\} \in \mathbb{Z}$ and $\{R\}:=R-[R] \in[0,1)$.

The following result shows that to define the function $\psi_{0}$ as a $\mathbb{Z}^{2}$-periodic function on $\mathbb{R}^{2}$, it is sufficient to restrict oneself to the domain $D$. (See Figure 4.)

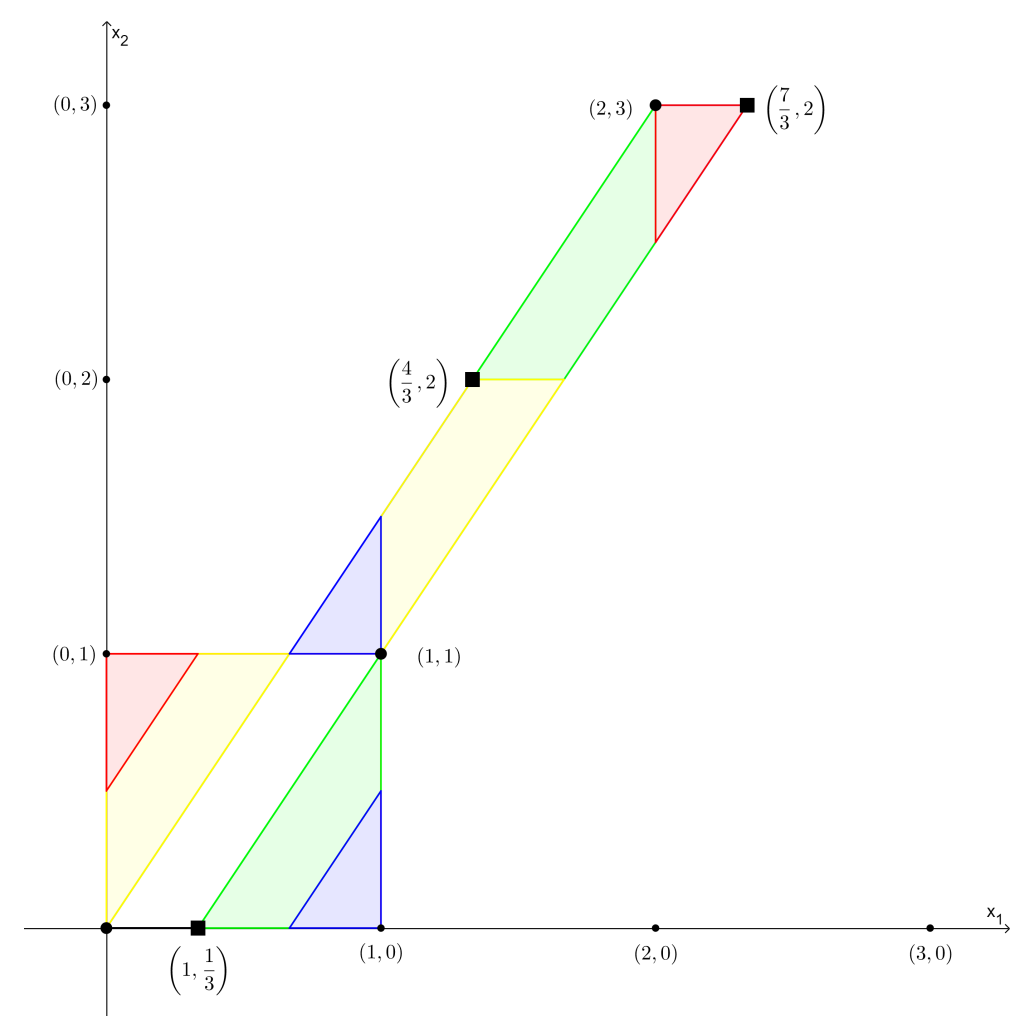

FIGURE 4. The domain $D$ for $(p, q)=(3,2)$.

Proposition 2.2. Let $p, q \in \mathbb{N}^{*}$ be relatively prime. Let

$$
D:=\left\{s\left(\frac{1}{p}, 0\right)+t\left(1, \frac{p}{q}\right), s \in[0,1), t \in[0, q)\right\} .
$$

Then for any $z \in \mathbb{R}^{2}$ there exists a unique pair $(x, y) \in D \times \mathbb{Z}^{2}$ such that $z=x+y$. Accordingly, $\pi(D)=\mathbb{T}^{2}$. 
Proof. It is sufficient to show that the function $f: D \rightarrow[0,1)^{2}$ defined by $f\left(x_{1}, x_{2}\right)=\left(\left\{x_{1}\right\},\left\{x_{2}\right\}\right)$ is bijective. Indeed, in such a case, we would have that for any given $\left(z_{1}, z_{2}\right) \in \mathbb{R}^{2}$, there would exist a unique $x=\left(x_{1}, x_{2}\right) \in D$ such that $x-\left(\left[x_{1}\right],\left[x_{2}\right]\right)=z-\left(\left[z_{1}\right],\left[z_{2}\right]\right) \in[0,1)^{2}$. Therefore, setting $y:=$ $\left(\left[z_{1}\right],\left[z_{2}\right]\right)-\left(\left[x_{1}\right],\left[x_{2}\right]\right) \in \mathbb{Z}^{2}$, we would get the desired result.

The fact that $f: D \rightarrow[0,1)^{2}$ is bijective means that the system

$$
\begin{aligned}
\left\{t+\frac{1}{p} s\right\} & =z_{1}, \\
\left\{\frac{p}{q} t\right\} & =z_{2},
\end{aligned}
$$

admits a unique solution $(s, t) \in[0,1) \times[0, q)$ for any $\left(z_{1}, z_{2}\right) \in[0,1)^{2}$.

Let us first show that $f: D \rightarrow[0,1)^{2}$ is one-to-one. Let $\left(s_{1}, s_{2}\right) \in[0,1)^{2}$ and $\left(t_{1}, t_{2}\right) \in[0, q)^{2}$ be such that

$$
f\left(t_{1}+\frac{s_{1}}{p}, \frac{t_{1} p}{q}\right)=f\left(t_{2}+\frac{s_{2}}{p}, \frac{t_{2} p}{q}\right)
$$

that is, $\left\{t_{1}+s_{1} / p\right\}=\left\{t_{2}+s_{2} / p\right\}$ and $\left\{t_{1} p / q\right\}=\left\{t_{2} p / q\right\}$. Then, setting $k_{1}:=\left[t_{1}+s_{1} / p\right]-\left[t_{2}+s_{2} / p\right] \in \mathbb{Z}$ and $k_{2}:=\left[t_{1} p / q\right]-\left[t_{2} p / q\right] \in \mathbb{Z}$, we obtain that

$$
\begin{aligned}
\left(t_{1}+\frac{s_{1}}{p}\right)-\left(t_{2}+\frac{s_{2}}{p}\right) & =\left[t_{1}+\frac{s_{1}}{p}\right]+\left\{t_{1}+\frac{s_{1}}{p}\right\}-\left(\left[t_{2}+\frac{s_{2}}{p}\right]+\left\{t_{2}+\frac{s_{2}}{p}\right\}\right)=k_{1}, \\
t_{1} \frac{p}{q}-t_{2} \frac{p}{q} & =\left[t_{1} \frac{p}{q}\right]+\left\{t_{1} \frac{p}{q}\right\}-\left(\left[t_{2} \frac{p}{q}\right]+\left\{t_{2} \frac{p}{q}\right\}\right)=k_{2} .
\end{aligned}
$$

It follows that

$$
\begin{aligned}
s_{1}-s_{2} & =p k_{1}-q k_{2}, \\
\left(t_{1}-t_{2}\right) p & =q k_{2} .
\end{aligned}
$$

Since $s_{1}-s_{2} \in(-1,1)$, the only possible value for $p k_{1}-q k_{2} \in \mathbb{Z}$ is zero. Thus $p k_{1}=q k_{2}$ and $s_{1}=s_{2}$. Since $p$ and $q$ are relatively prime, we infer that we can write $k_{2}=l p$ for some $l \in \mathbb{Z}$. Il follows that $\left(t_{1}-t_{2}\right) p=l p q$, and hence $t_{1}-t_{2}=l q$. But $t_{1}-t_{2} \in(-q, q)$, so we infer that $t_{1}=t_{2}$.

Let us prove that $f: D \rightarrow[0,1)^{2}$ is onto. Given $\left(z_{1}, z_{2}\right) \in[0,1)^{2}$, we aim to find $s \in[0,1)$ and $t \in[0, q)$ such that system (2.3)-(2.4) is satisfied. Note that (2.3)-(2.4) is equivalent to the existence of some numbers $k_{1}, k_{2} \in \mathbb{Z}$ such that

$$
\begin{aligned}
t+\frac{s}{p} & =z_{1}+k_{1}, \\
t \frac{p}{q} & =z_{2}+k_{2} .
\end{aligned}
$$

Eliminating $t$ from (2.7), we arrive at the equivalent system

$$
\begin{aligned}
s & =\left(p z_{1}-q z_{2}\right)+\left(p k_{1}-q k_{2}\right), \\
p t & =q z_{2}+q k_{2} .
\end{aligned}
$$

Since $p k_{1}-q k_{2} \in \mathbb{Z}$, we have to pick $s=\left\{p z_{1}-q z_{2}\right\}$. Next, the equation (2.9) will be satisfied if and only if

$$
p k_{1}-q k_{2}=-\left[p z_{1}-q z_{2}\right]
$$


Since $p$ and $q$ are relatively prime, there exists by Bezout's identity a pair $(a, b) \in \mathbb{Z}^{2}$ such that $a p+b q=$ 1 . Let $l:=-\left[p z_{1}-q z_{2}\right] \in \mathbb{Z}$ and

$$
k_{1}:=a l+r q, \quad k_{2}:=-b l+r p,
$$

where $r \in \mathbb{Z}$ remains to be defined. Then $k_{1}, k_{2} \in \mathbb{Z}$ and (2.11) is satisfied. It remains to fulfill (2.10), which can be rewritten $t / q=\left(z_{2}-b l\right) / p+r$, with the constraint $t \in[0, q)$. Picking $r:=-\left[\left(z_{2}-b l\right) / p\right]$ and $t:=q\left(\left(z_{2}-b l\right) / p+r\right) \in[0, q)$, we infer that (2.10) is also satisfied. The proof of Proposition 2.2 is complete.

Proposition 2.3. Let $c=(1, p / q)$, where $p, q \in \mathbb{N}^{*}$ are relatively prime. Let $\omega_{0} \subset \mathbb{T}^{2}$ be a nonempty open set such that $\pi([0,1 / p] \times\{0\}) \subset \omega_{0}$, and let $\tau \in(0,1]$ be given. Then there exists a nonnegative function $\psi_{0} \in C^{\infty}\left(\mathbb{T}^{2}\right)$ such that

$$
\begin{aligned}
2 \tau \leq c \cdot \nabla \psi_{0} \leq 2(q+\tau) & \text { in } \quad \mathbb{T}^{2} \backslash \omega_{0}, \\
c \cdot \nabla\left(c \cdot \nabla \psi_{0}\right)=2 & \text { in } \quad \mathbb{T}^{2} \backslash \omega_{0} .
\end{aligned}
$$

Proof. Our goal is to construct a suitable function $\psi_{0}$ in $D$ and then to extend it to $\mathbb{R}^{2}$ as a smooth $\mathbb{Z}^{2}$-periodic function. Therefore, it is important to determine what pairs of points on $\partial D$ are identified when viewed as points in $\mathbb{T}^{2}$, that is, modulo $\mathbb{Z}^{2}$. We first focus on the point $(1 / p, 0) \in \partial D$ and determine all the points on $\partial D$ that can be identified with it. (See Figure 5.)

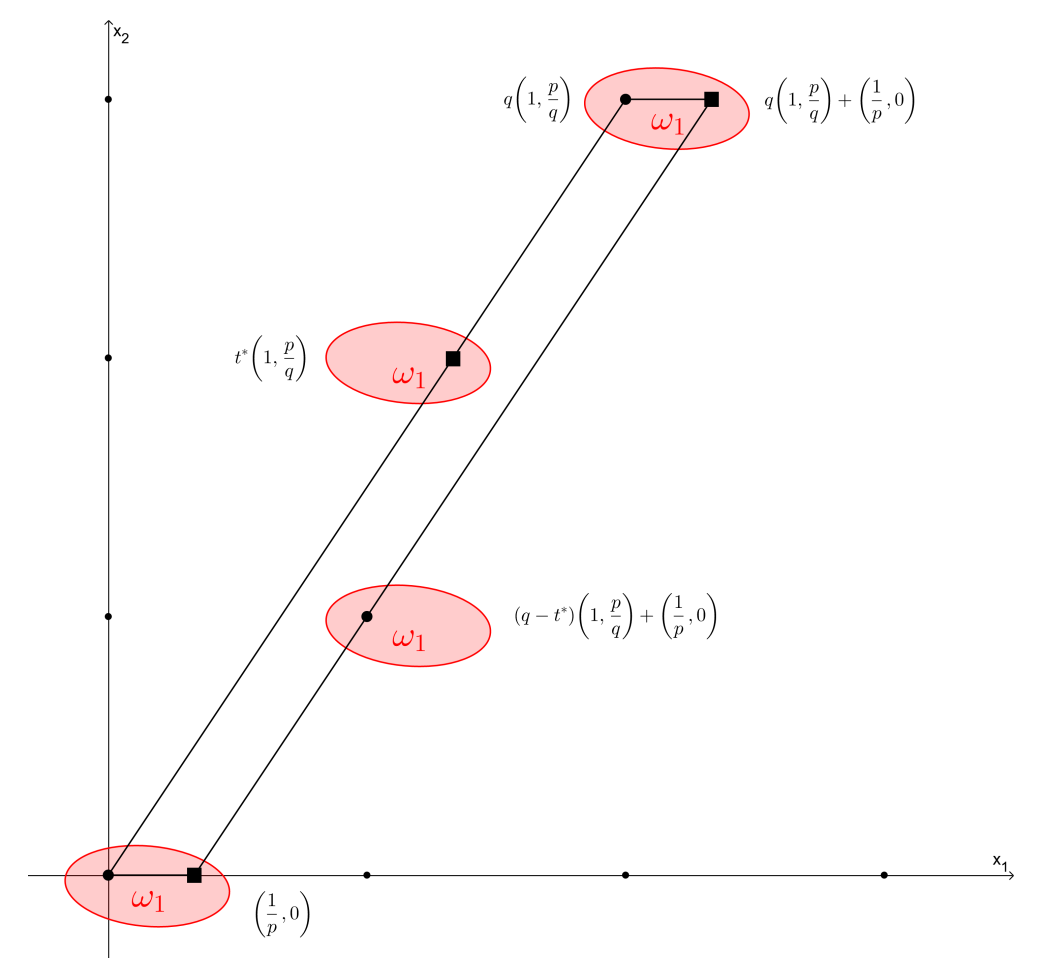

Figure 5. The domain $D$ and the open set $\omega_{1}$ for $(p, q)=(3,2)$. Note that $t^{*}=4 / 3$. 
Lemma 2.1. There exists a unique number $t^{*} \in[0, q)$ such that $t^{*}(1, p / q)-(1 / p, 0) \in \mathbb{Z}^{2}$.

Proof of Lemma 2.1. Since $p$ and $q$ are relatively prime, by Bézout's identity there exists a pair $(a, b) \in \mathbb{Z}^{2}$ such that $a p+b q=1$. Since $(a-q m) p+(b+m p) q=1$ for any $m \in \mathbb{Z}$, we may without loss of generality assume that $b \in[0, p)$. Set $t^{*}=b q / p \in[0, q)$. Then $t^{*}-1 / p=-a \in \mathbb{Z}$ and $t^{*} p / q=b \in \mathbb{Z}$, so that $t^{*}(1, p / q)-(1 / p, 0) \in \mathbb{Z}^{2}$. On the other hand, it follows from Proposition 2.2 that there exists a unique pair $(x, y) \in D \times \mathbb{Z}^{2}$ such that $(1 / p, 0)=x+y$. Decomposing $x$ as $x=s(1 / p, 0)+t(1, p / q)$, we infer that there exists a unique pair $(s, t) \in[0,1) \times[0, q)$ such that

$$
(1 / p, 0)-[s(1 / p, 0)+t(1, p / q)] \in \mathbb{Z}^{2} .
$$

This yields $(s, t)=\left(0, t^{*}\right)$ and the uniqueness of $t^{*}$.

Let us go back to the proof of Proposition 2.3. We start with a claim, which is a direct consequence of Lemma 2.1.

CLAim 1. For every $t \in\left[0, q-t^{*}\right],\left(t+t^{*}\right)(1, p / q) \in \partial D$ is identified in $\mathbb{T}^{2}$ with $t(1, p / q)+(1 / p, 0) \in \partial D$. For every $t \in\left[q-t^{*}, q\right],\left(t-\left(q-t^{*}\right)\right)(1, p / q) \in \partial D$ is identified with $t(1, p / q)+(1 / p, 0) \in \partial D$.

Since $\pi([0,1 / p] \times\{0\}) \subset \omega_{0}$, we can pick two open sets $\omega_{1}, \omega_{2}$ in $\mathbb{T}^{2}$ such that $\pi([0,1 / p] \times\{0\}) \subset \omega_{1}$ and $\overline{\omega_{1}} \subset \omega_{2} \subset \overline{\omega_{2}} \subset \omega_{0}$. Since, by Lemma $2.1,\left(q-t^{*}\right)(1, p / q)+(1 / p, 0) \in \mathbb{Z}^{2}$ is identified with $(0,0)$, and since the open set $\omega_{1}$ contains $(0,0)$, we can select a number $\varepsilon \in(0,1)$ such that for every $s \in[1-\varepsilon, 1]$ we have that $\left(q-t^{*}\right)(1, p / q)+s(1 / p, 0) \in \omega_{1}$.

We need to introduce two cut-off functions. Pick $\chi \in C^{\infty}\left(\mathbb{T}^{2}\right)$ with $0 \leq \chi \leq 1$ such that

$$
\chi(x)= \begin{cases}1 & \text { if } x \in \mathbb{T}^{2} \backslash \omega_{2}, \\ 0 & \text { if } x \in \overline{\omega_{1}},\end{cases}
$$

and $\eta \in C^{\infty}([0,1])$ with $0 \leq \eta \leq 1$ and such that

$$
\eta(s)= \begin{cases}1 & \text { if } s \leq 1-\varepsilon \\ 0 & \text { if } s \geq 1-\frac{\varepsilon}{2}\end{cases}
$$

Introduce the functions $\psi_{1}, \psi_{2}:[0, q] \rightarrow \mathbb{R}$ defined by

$$
\begin{aligned}
& \psi_{1}(t)=(t+\tau)^{2}, \quad t \in[0, q], \\
& \psi_{2}(t)= \begin{cases}\left(t+t^{*}+\tau\right)^{2}, & \text { if } t \in\left[0, q-t^{*}\right], \\
\left(t+t^{*}-q+\tau\right)^{2}, & \text { if } t \in\left(q-t^{*}, q\right] .\end{cases}
\end{aligned}
$$

Note that

$$
\psi_{2}(t)= \begin{cases}\psi_{1}\left(t+t^{*}\right), & \text { if } t \in\left[0, q-t^{*}\right) \\ \psi_{1}\left(t-\left(q-t^{*}\right)\right), & \text { if } t \in\left(q-t^{*}, q\right]\end{cases}
$$

Finally, taking into account the fact that any point $x \in D$ can be written in a unique way as $x=t(1, p / q)+$ $s(1 / p, 0)$ with $(s, t) \in[0,1) \times[0, q)$, we define the function $\psi_{0}: D \rightarrow \mathbb{R}_{+}$by

$\psi_{0}(t(1, p / q)+s(1 / p, 0))=\left[\eta(s) \psi_{1}(t)+(1-\eta(s)) \psi_{2}(t)\right] \chi(t(1, p / q)+s(1 / p, 0)), \quad(s, t) \in[0,1) \times[0, q)$.

Using Proposition 2.2, we can extend $\psi_{0}$ to $\mathbb{R}^{2}=D+\mathbb{Z}^{2}$ by setting

$$
\psi_{0}(x+y)=\psi_{0}(x), \quad \forall x \in D, \forall y \in \mathbb{Z}^{2} .
$$

The function $\psi_{0}$ being $\mathbb{Z}^{2}$-periodic, it can be viewed as a map from $\mathbb{T}^{2}$ to $\mathbb{R}$. It turns that $\psi_{0}$ is smooth. 
Claim 2. $\psi_{0} \in C^{\infty}\left(\mathbb{T}^{2}\right)$.

Indeed, the function $\psi_{0}$ is smooth:

(i) in the interior of $\pi(D)$, namely in the set $\{\pi(t(1, p / q)+s(1 / p, 0)) ;(s, t) \in(0,1) \times(0, q)\}$;

(ii) in a neighborhood of the segment $[0,1 / p] \times\{0\}$ thanks to the definitions of $\omega_{1}$ and $\chi$;

(iii) in a neighborhood of the segment $\left\{\left(q-t^{*}\right)(1, p / q)+s(1 / p, 0) ; s \in[1-\varepsilon, 1]\right\}$ thanks to the definition of $\omega_{1}, \chi$ and $\eta$ (even if the function $\psi_{2}$ is discontinuous at $t=q-t^{*}$ );

(iv) in a neighborhood of the segments $\{t(1, p / q) ; t \in[0, q]\}$ and $\{(1 / p, 0)+t(1, p / q) ; t \in[0, q]\}$ thanks to Claim 1 and to the definitions of $\psi_{1}$ and $\psi_{2}$.

It remains to check that $\psi_{0}$ fulfills (2.12) and (2.13). But we notice that for $x=s(1 / p, 0)+t(1, p / q) \in$ $\mathbb{T}^{2} \backslash \overline{\omega_{2}}, \chi(x)=1$ and

$$
\begin{aligned}
c \cdot \nabla \psi_{0}(x) & =\frac{\partial}{\partial t}\left[\psi_{0}(s(1 / p, 0)+t(1, p / q))\right]=\eta(s) \psi_{1}^{\prime}(t)+(1-\eta(s)) \psi_{2}^{\prime}(t) \in[2 \tau, 2(q+\tau)], \\
c \cdot \nabla\left(c \cdot \nabla \psi_{0}\right)(x) & =\frac{\partial^{2}}{\partial t^{2}}\left[\psi_{0}(s(1 / p, 0)+t(1, p / q))\right]=\eta(s) \psi_{1}^{\prime \prime}(t)+(1-\eta(s)) \psi_{2}^{\prime \prime}(t)=2 .
\end{aligned}
$$

Noting that $\mathbb{T}^{2} \backslash \omega_{0} \subset \mathbb{T}^{2} \backslash \overline{\omega_{2}}$, we infer that (2.12)-(2.13) are fulfilled. The proof of Proposition 2.3 is complete.

(iii) $c=(1, \zeta), \zeta \in \mathbb{R}_{+} \backslash \mathbb{Q}$.

Before performing the construction of the function $\psi_{0}$, we first prove Proposition 1.2.

Proof of Proposition 1.2: If there does not exist $T>0$ such that $\omega_{0}+[0, T] c=\mathbb{T}^{2}$, then for any $n \in \mathbb{N}^{*}$ we can pick some $y_{n} \in \mathbb{T}^{2}$ such that

$$
y_{n} \notin \omega_{0}+[0, n] c .
$$

As $\mathbb{T}^{2}$ is compact, we can extract a subsequence $y_{n_{k}} \rightarrow y$ for some $y \in \mathbb{T}^{2}$. Pick $x \in \mathbb{T}^{2}$ and $\varepsilon>0$ such that $B(x, \varepsilon) \subset \omega_{0}$, and let $z=\left(z_{1}, z_{2}\right)=y-x$. As the subgroup $\{\zeta p+q ; p, q \in \mathbb{Z}\}$ is dense in $\mathbb{R}$ (for $\zeta \notin \mathbb{Q}$ ), we infer the existence of a pair $(p, q) \in \mathbb{Z}^{2}$ such that $\left|z_{2}-\zeta z_{1}-(\zeta p+q)\right|<\varepsilon / 4$. As $\zeta \in \mathbb{R}_{+} \backslash \mathbb{Q}=\mathcal{J}_{1 / \sqrt{5}}$, one may find a pair $\left(p^{\prime}, q^{\prime}\right) \in \mathbb{N}^{2}$ (with $q^{\prime}$ as large as desired) such that $\left|\zeta-\frac{p^{\prime}}{q^{\prime}}\right| \leq\left(\sqrt{5} q^{\prime 2}\right)^{-1}$. In particular, we can impose both conditions $q^{\prime} \geq\left|z_{1}+p\right|+1$ and $\left(\sqrt{5} q^{\prime}\right)^{-1}<\varepsilon / 4$. Set $t=z_{1}+p+q^{\prime}>0$. Then

$$
\begin{aligned}
\left|\left(z_{1}, z_{2}\right)-t(1, \zeta)-\left(p+q^{\prime}, q-p^{\prime}\right)\right| & =\left|z_{2}-\left(z_{1}+p+q^{\prime}\right) \zeta-q+p^{\prime}\right| \\
& \leq\left|z_{2}-\zeta z_{1}-(\zeta p+q)\right|+\left|p^{\prime}-q^{\prime} \zeta\right| \\
& \leq \frac{\varepsilon}{2} .
\end{aligned}
$$

It follows that for $n$ large enough, $\left|y_{n}-x-t c-\left(p+q^{\prime}, q-p^{\prime}\right)\right|<\varepsilon$, that is $y_{n} \in B(x, \varepsilon)+t c$, contradicting the assumption.

Let us proceed with the construction of the function $\psi_{0}$ when $c=(1, \zeta)$ with $\zeta \in \mathbb{R} \backslash \mathbb{Q}_{+}$. The idea is to use the function $\psi_{0}$ constructed above for $\hat{c}=\left(1, \frac{p}{q}\right)$ when $p / q$ is a "good" rational approximation of $\zeta$, namely satisfying (1.25). Note that in this case $|q \zeta-p| \leq \delta / q$, so that

$$
p \geq q \zeta-\frac{\delta}{q} \geq \frac{1}{2} \zeta q
$$


provided that $A \gg 1$. (Recall that $q \geq A$.) Moreover

$$
\left|\frac{q}{p} \zeta-1\right| \leq \frac{\delta}{p q} \leq \frac{2 \delta}{\zeta q^{2}}
$$

provided that $A \gg 1$.

Proposition 2.4. Assume that $c=(1, \zeta)$, where $\zeta \in \mathcal{J}_{\delta}$ for some $\delta>0$. Let $\omega_{0}$ be an open neighbourhood of 0 . Pick $A>0$ and a pair $(p, q)$ with $q \geq A$ and such that (1.22), (1.25), (2.17) and (2.18) are satisfied, and such that

$$
\frac{1}{p}<\operatorname{dist}\left(0, \mathbb{T}^{2} \backslash \omega_{0}\right) .
$$

Let $\hat{c}=\left(1, \frac{p}{q}\right)$. Then there exists a function $\psi_{0} \in C^{\infty}\left(\mathbb{T}^{2}\right)$ such that

$$
\begin{array}{rcc}
\nabla \psi_{0}(x) \neq 0 & \text { in } & \mathbb{T}^{2} \backslash \omega_{0}, \\
\left|(c-\hat{c}) \cdot \nabla \psi_{0}\right| \leq 8 \delta q+f_{1}(\delta, \zeta) & \text { in } \quad & \mathbb{T}^{2} \backslash \omega_{0}, \\
\left|c \cdot \nabla\left(c \cdot \nabla \psi_{0}\right)-2\right| \leq f_{2}(\delta, \zeta) & \text { in } \quad & \mathbb{T}^{2} \backslash \omega_{0},
\end{array}
$$

where

$$
\begin{aligned}
f_{1}(\delta, \zeta) & :=\left(\frac{8}{\zeta}+16\right) \delta \\
f_{2}(\delta, \zeta) & :=\left(32+\frac{8}{\zeta}\right) \delta+\left(549+\frac{64}{\zeta}+\frac{8}{\zeta^{2}}\right) \delta^{2} .
\end{aligned}
$$

Proof. It follows from (2.19) that $\left[-\frac{1}{p}, \frac{1}{p}\right] \times\{0\} \subset \omega_{0}$. We pick two open sets $\omega_{1}, \omega_{2}$ with

$$
\left[-\frac{1}{p}, \frac{1}{p}\right] \times\{0\} \subset \omega_{1} \subset \overline{\omega_{1}} \subset \omega_{2} \subset \overline{\omega_{2}} \subset \omega_{0} .
$$

Let the functions $\chi, \eta, \psi_{1}, \psi_{2}$ and $\psi_{0}$ be as in the proof of Proposition 2.3 for $\hat{c}=\left(1, \frac{p}{q}\right)$ and $\varepsilon=1 / 2$. Note that it is easy to construct a function $\eta$ as above with the following explicit bounds

$$
\left\|\eta^{\prime}\right\|_{L^{\infty}(\mathbb{R})} \leq 2^{3}, \quad\left\|\eta^{\prime \prime}\right\|_{L^{\infty}(\mathbb{R})} \leq 2^{7}\left(\int_{\mathbb{R}} \exp \left(1-\frac{1}{1-y^{2}}\right) d y\right)^{-1} \leq 183 .
$$

Clearly, (2.20) follows at once from (2.12). Set $\hat{d}=\left(\frac{1}{p}, 0\right)$. Then

$$
c=(1, \zeta)=\frac{q}{p} \zeta \hat{c}+(p-q \zeta) \hat{d} .
$$

From the definition of $\psi_{0},(2.17),(2.18)$ and (2.25), we obtain that for $x=s \hat{d}+t \hat{c} \in \mathbb{T}^{2} \backslash \overline{\omega_{2}}$ (where $s \in[0,1), t \in[0, q))$

$$
\begin{aligned}
(c-\hat{c}) \cdot \nabla \psi_{0}(x) & =\left(\frac{q}{p} \zeta-1\right) \hat{c} \cdot \nabla \psi_{0}(x)+(p-q \zeta) \hat{d} \cdot \nabla \psi_{0}(x) \\
& =\left(\frac{q}{p} \zeta-1\right) \frac{\partial}{\partial t}\left[\psi_{0}(s \hat{d}+t \hat{c})\right]+(p-q \zeta) \frac{\partial}{\partial s}\left[\psi_{0}(s \hat{d}+t \hat{c})\right] \\
& =\left(\frac{q}{p} \zeta-1\right)\left(\eta(s) \psi_{1}^{\prime}(t)+(1-\eta(s)) \psi_{2}^{\prime}(t)\right)+(p-q \zeta) \eta^{\prime}(s)\left(\psi_{1}(t)-\psi_{2}(t)\right) .
\end{aligned}
$$


Recall that for $i=1,2$,

$$
\psi_{i}(t) \in\left[\tau^{2},(q+\tau)^{2}\right], \quad \psi_{i}^{\prime}(t) \in[2 \tau, 2(q+\tau)], \quad \psi_{i}^{\prime \prime}(t)=2 .
$$

It follows that

$$
\begin{aligned}
\left|(c-\hat{c}) \cdot \nabla \psi_{0}(x)\right| & \leq\left|\frac{q}{p} \zeta-1\right| \cdot 2(q+\tau)+|p-q \zeta| \cdot 8 \cdot\left(q^{2}+2 q \tau\right) \\
& \leq \frac{2 \delta}{\zeta q^{2}} \cdot 2(q+\tau)+\frac{8 \delta}{q}\left(q^{2}+2 q \tau\right) \\
& \leq \delta\left(8 q+\frac{8}{\zeta}+16\right)
\end{aligned}
$$

for $q \geq 1$ and $\tau \leq 1$. We can write

$$
\begin{aligned}
c \cdot \nabla\left(c \cdot \nabla \psi_{0}\right)(x)-\hat{c} \cdot \nabla\left(\hat{c} \cdot \nabla \psi_{0}\right) & =c \cdot \nabla\left((c-\hat{c}) \cdot \nabla \psi_{0}\right)(x)+(c-\hat{c}) \cdot \nabla\left(\hat{c} \cdot \nabla \psi_{0}\right)(x) \\
& =: I_{1}+I_{2} .
\end{aligned}
$$

Then

$$
\begin{aligned}
I_{1}= & \frac{q}{p} \zeta \frac{\partial}{\partial t}\left(\left[(c-\hat{c}) \cdot \nabla \psi_{0}\right](s \hat{d}+t \hat{c})\right)+(p-q \zeta) \frac{\partial}{\partial s}\left(\left[(c-\hat{c}) \cdot \nabla \psi_{0}\right](s \hat{d}+t \hat{c})\right) \\
= & \frac{q}{p} \zeta\left[\left(\frac{q}{p} \zeta-1\right)\left(\eta(s) \psi_{1}^{\prime \prime}(t)+(1-\eta(s)) \psi_{2}^{\prime \prime}(t)\right)+(p-q \zeta) \eta^{\prime}(s)\left(\psi_{1}^{\prime}(t)-\psi_{2}^{\prime}(t)\right)\right] \\
& \quad+(p-q \zeta)\left[\left(\frac{q}{p} \zeta-1\right) \eta^{\prime}(s)\left(\psi_{1}^{\prime}(t)-\psi_{2}^{\prime}(t)\right)+(p-q \zeta) \eta^{\prime \prime}(s)\left(\psi_{1}(t)-\psi_{2}(t)\right)\right] .
\end{aligned}
$$

Thus

$$
\begin{aligned}
\left|I_{1}\right| \leq & \left|\frac{q}{p} \zeta\right|\left[\left|\frac{q}{p} \zeta-1\right| \cdot 2+|p-q \zeta| \cdot 2^{3} \cdot 2 q\right] \\
& +|p-q \zeta|\left[\left|\frac{q}{p} \zeta-1\right| \cdot 8 \cdot 2 q+|p-q \zeta| \cdot 183 \cdot\left(q^{2}+2 \tau q\right)\right] \\
\leq & \left(1+\frac{2 \delta}{\zeta q^{2}}\right)\left(\frac{2 \delta}{\zeta q^{2}} \cdot 2+\frac{\delta}{q} \cdot 16 q\right) \\
& \quad+\frac{\delta}{q}\left(\frac{2 \delta}{\zeta q^{2}} \cdot 16 q+\frac{\delta}{q} \cdot 183 \cdot\left(q^{2}+2 \tau q\right)\right) \\
\leq & 16 \delta+183 \delta^{2}+\frac{32 \delta^{2}}{\zeta q^{2}}+\frac{16 \delta^{2}}{q}+\left(\frac{32 \delta^{2}}{\zeta}+\frac{4 \delta}{\zeta}\right) \frac{1}{q^{2}}+\frac{8 \delta^{2}}{\zeta^{2} q^{4}}
\end{aligned}
$$

where we used the fact that $\tau \in[0,1]$. On the other hand, we have that

$$
\begin{aligned}
I_{2} & =\left(\frac{q}{p} \zeta-1\right) \hat{c} \cdot \nabla\left(\hat{c} \cdot \nabla \psi_{0}\right)+(p-q \zeta) \hat{d} \cdot \nabla\left(\hat{c} \cdot \nabla \psi_{0}\right) \\
& =\left(\frac{q}{p} \zeta-1\right) \frac{\partial^{2}}{\partial t^{2}}\left[\psi_{0}(s \hat{d}+t \hat{c})\right]+(p-q \zeta) \cdot \frac{\partial}{\partial s}\left(\frac{\partial}{\partial t}\left[\psi_{0}(s \hat{d}+t \hat{c})\right]\right) \\
& =\left(\frac{q}{p} \zeta-1\right)\left(\eta(s) \psi_{1}^{\prime \prime}(t)+(1-\eta(s)) \psi_{2}^{\prime \prime}(t)\right)+(p-q \zeta) \eta^{\prime}(s)\left(\psi_{1}^{\prime}(t)-\psi_{2}^{\prime}(t)\right) .
\end{aligned}
$$


It follows that

$$
\begin{aligned}
\left|I_{2}\right| & \leq\left|\frac{q}{p} \zeta-1\right| \cdot 2+|p-q \zeta| \cdot 8 \cdot 2 q \\
& \leq \frac{2 \delta}{\zeta q^{2}} \cdot 2+\frac{\delta}{q} \cdot 16 q=16 \delta+\frac{4 \delta}{\zeta q^{2}} .
\end{aligned}
$$

We infer that

$$
\begin{aligned}
\left|c \cdot \nabla\left(c \cdot \nabla \psi_{0}\right)-2\right|= & \left|c \cdot \nabla\left(c \cdot \nabla \psi_{0}\right)-\hat{c} \cdot \nabla\left(\hat{c} \cdot \nabla \psi_{0}\right)\right| \\
\leq & \left|I_{1}\right|+\left|I_{2}\right| \\
\leq & 32 \delta+183 \delta^{2} \\
& \quad+366 \frac{\delta^{2}}{q}+\left(\frac{8 \delta}{\zeta}+\frac{64 \delta^{2}}{\zeta}\right) \frac{1}{q^{2}}+\frac{8 \delta^{2}}{\zeta^{2} q^{4}} \\
\leq & \left(32+\frac{8}{\zeta}\right) \delta+\left(549+\frac{64}{\zeta}+\frac{8}{\zeta^{2}}\right) \delta^{2} .
\end{aligned}
$$

\section{CARLEMAN ESTIMATES}

In this section, we derive a Carleman estimate for a transport equation and a Carleman estimate for a heat equation with the same weights.

Let $\psi_{0} \in C^{\infty}\left(\mathbb{T}^{2}\right), K, \widetilde{K} \in(0,+\infty)$ and $t_{0} \in[0, T]$ (to be chosen later on), and set

$$
\psi(x, t)=\psi_{0}(x)-K\left(t-t_{0}\right)^{2}+\widetilde{K}, \quad(x, t) \in \mathbb{T}^{2} \times[0, T] .
$$

By picking $\widetilde{K}$ large enough, we can assume that

$$
\psi(x, t)>\frac{3}{4}\|\psi\|_{L^{\infty}\left(\mathbb{T}^{2} \times(0, T)\right)}, \quad \forall x \in \mathbb{T}^{2}, \forall t \in[0, T] .
$$

For given $\sigma \in(0, \min (1, T / 2))$, we introduce some function $g \in C^{\infty}(0, T)$ such that

$$
g(t)= \begin{cases}\frac{1}{t} & \text { for } 0<t<\frac{\sigma}{2} \\ \text { strictly decreasing } & \text { for } 0<t \leq \sigma \\ 1 & \text { for } \sigma \leq t \leq \frac{T}{2} \\ g(T-t) & \text { for } \frac{T}{2}<t \leq T\end{cases}
$$

We define the weights

$$
\begin{aligned}
& \varphi(x, t):=g(t)\left(e^{\frac{3}{2} \lambda\|\psi\|_{L^{\infty}\left(\mathbb{T}^{2} \times(0, T)\right)}}-e^{\lambda \psi(x, t)}\right), \\
& \theta(x, t):=g(t) e^{\lambda \psi(x, t)},
\end{aligned}
$$

where $\lambda>0$ is a parameter.

The Carleman estimate for the transport equation is given in the following proposition. 
Proposition 3.1. Let $c=(1, \zeta)$ with $\zeta \in \mathbb{R}_{+}$. Let $T>0, \sigma \in(0, \min (1, T / 2))$ and $K, \tilde{K}>0$. Assume that the function $\psi_{0} \in C^{\infty}\left(\mathbb{T}^{2}\right)$ satisfies for some nonempty open set $\omega_{0} \subset \mathbb{T}^{2}$ and some number $\hat{\delta}>0$

$$
\begin{array}{rlrl}
\nabla \psi_{0}(x) & \neq 0, & & \forall x \in \mathbb{T}^{2} \backslash \omega_{0}, \\
-2 K\left(t-t_{0}\right)-c \cdot \nabla \psi_{0}(x) \geq 0, & & \forall x \in \mathbb{T}^{2} \backslash \omega_{0}, \forall t \in(0, \sigma), \\
-2 K\left(t-t_{0}\right)-c \cdot \nabla \psi_{0}(x) \leq 0, & & \forall x \in \mathbb{T}^{2} \backslash \omega_{0}, \forall t \in(T-\sigma, T), \\
c \cdot \nabla\left(c \cdot \nabla \psi_{0}\right)(x)-2 K \geq \hat{\delta}, & & \forall x \in \mathbb{T}^{2} \backslash \omega_{0} .
\end{array}
$$

Let the functions $\psi, g, \varphi$, and $\theta$ be as in (3.1)-(3.5). Then there exist some constants $\lambda_{0} \geq 1, s_{0} \geq 1$ and $C_{0}>0$ such that for all $\lambda \geq \lambda_{0}$, all $s \geq s_{0}$ and all $w \in L^{2}\left(\mathbb{T}^{2} \times(0, T)\right)$ with $-w_{t}+c \cdot \nabla w \in L^{2}\left(\mathbb{T}^{2} \times(0, T)\right)$, we have

$$
\begin{aligned}
& \int_{0}^{T} \int_{\mathbb{T}^{2}} \lambda s \theta|w|^{2} e^{-2 s \varphi} d x d t \\
& \quad \leq C_{0}\left(\int_{0}^{T} \int_{\mathbb{T}^{2}}\left|-w_{t}+c \cdot \nabla w\right|^{2} e^{-2 s \varphi} d x d t+\int_{0}^{T} \int_{\omega_{0}} \lambda s \theta\left(s \frac{\left|g^{\prime}\right|}{g} \varphi+1\right)|w|^{2} e^{-2 s \varphi} d x d t\right) .
\end{aligned}
$$

Proof. Assume first that $w \in H^{1}\left(\mathbb{T}^{2} \times(0, T)\right)$. Let $z=e^{-s \varphi} w$ and $P w=-w_{t}+c \cdot \nabla w$. Then

$$
\begin{aligned}
e^{-s \varphi} P w & :=e^{-s \varphi} P\left(e^{s \varphi} z\right)=-\left(s \varphi_{t} z+z_{t}\right)+c \cdot(s z \nabla \varphi+\nabla z) \\
& =P_{1} z+P_{2} z
\end{aligned}
$$

with

$$
\begin{aligned}
P_{1} z & :=-s \varphi_{t} z-z_{t}+c \cdot \nabla z \\
P_{2} z & :=s(c \cdot \nabla \varphi) z .
\end{aligned}
$$

It follows that

$$
\left\|e^{-s \varphi} P w\right\|^{2}=\left\|P_{1} z\right\|^{2}+\left\|P_{2} z\right\|^{2}+2\left(P_{1} z, P_{2} z\right)
$$

where $(f, g)=\int_{0}^{T} \int_{\mathbb{T}^{2}} f g d x d t,\|f\|^{2}=(f, f)$. In the sequel, $\int_{0}^{T} \int_{\mathbb{T}^{2}} f(x, t) d x d t$ is denoted $\iint f$ for the sake of shortness.

The idea is to expand $\left(P_{1} z, P_{2} z\right)$ and next to use the (nonnegative) terms $\left\|P_{1} z\right\|^{2}$ and $\left\|P_{2} z\right\|^{2}$ to balance the "bad terms" in $\left(P_{1} z, P_{2} z\right)$. Using integration by parts, we obtain

$$
\begin{aligned}
\left(P_{1} z, P_{2} z\right) & =-s^{2} \iint(c \cdot \nabla \varphi) \varphi_{t} z^{2}-s \iint(c \cdot \nabla \varphi) z z_{t}+\frac{s}{2} \iint(c \cdot \nabla \varphi) c \cdot \nabla z^{2} \\
& =-s^{2} \iint(c \cdot \nabla \varphi) \varphi_{t} z^{2}+\frac{s}{2} \iint\left(c \cdot \nabla \varphi_{t}\right) z^{2}-\frac{s}{2} \iint c \cdot \nabla(c \cdot \nabla \varphi) z^{2}
\end{aligned}
$$


Easy calculations show that

$$
\begin{aligned}
\nabla \varphi & =-\lambda \theta \nabla \psi, \\
\varphi_{t} & =\frac{g^{\prime}}{g} \varphi-\lambda \theta \psi_{t}, \\
\nabla \varphi_{t} & =-\lambda \frac{g^{\prime}}{g} \theta \nabla \psi-\lambda^{2} \theta \psi_{t} \nabla \psi, \\
\nabla(c \cdot \nabla \varphi) & =-\lambda(c \cdot \nabla \psi) \nabla \theta-\lambda \theta \nabla(c \cdot \nabla \psi), \\
c \cdot \nabla(c \cdot \nabla \varphi) & =-\lambda^{2}(c \cdot \nabla \psi)^{2} \theta-\lambda \theta c \cdot \nabla(c \cdot \nabla \psi), \\
\nabla \psi_{t} & =0 .
\end{aligned}
$$

We infer that

$$
\begin{aligned}
\left(P_{1} z, P_{2} z\right)=\iint & {\left[s^{2} \lambda(c \cdot \nabla \psi) \theta\left(\frac{g^{\prime}}{g} \varphi-\lambda \theta \psi_{t}\right)-\frac{s}{2}\left(\lambda \frac{g^{\prime}}{g} \theta c \cdot \nabla \psi+\lambda^{2} \theta \psi_{t} c \cdot \nabla \psi\right)\right.} \\
& \left.+\frac{s}{2}\left(\lambda^{2}(c \cdot \nabla \psi)^{2} \theta+\lambda \theta c \cdot \nabla(c \cdot \nabla \psi)\right)\right] z^{2}
\end{aligned}
$$

Next

$$
\left\|P_{2} z\right\|^{2}=s^{2} \lambda^{2} \iint(c \cdot \nabla \psi)^{2} \theta^{2} z^{2}
$$

and

$$
0 \leq\left\|P_{1} z-s \lambda \psi_{t} \theta z\right\|^{2}=\left\|P_{1} z\right\|^{2}+s^{2} \lambda^{2}\left\|\psi_{t} \theta z\right\|^{2}-2 s \lambda\left(P_{1} z, \psi_{t} \theta z\right)
$$

But

$$
\begin{aligned}
\left(P_{1} z, \psi_{t} \theta z\right) & =-s \iint \varphi_{t} \psi_{t} \theta z^{2}-\iint \psi_{t} \theta z_{t} z+\iint(c \cdot \nabla z) \psi_{t} \theta z \\
& =-s \iint\left(\frac{g^{\prime}}{g} \varphi-\lambda \theta \psi_{t}\right) \psi_{t} \theta z^{2}+\iint\left(\psi_{t t} \theta+\psi_{t} \frac{g^{\prime}}{g} \theta+\lambda \theta \psi_{t}^{2}\right) \frac{z^{2}}{2}-\iint \lambda \psi_{t}(c \cdot \nabla \psi) \theta \frac{z^{2}}{2} .
\end{aligned}
$$

Therefore

$$
\begin{aligned}
2 s \lambda\left(P_{1} z, \psi_{t} \theta z\right)= & 2 s^{2} \lambda^{2} \iint \psi_{t}^{2} \theta^{2} z^{2}-2 s^{2} \lambda \iint \frac{g^{\prime}}{g} \varphi \psi_{t} \theta z^{2} \\
& +s \lambda \iint\left(\psi_{t t} \theta+\frac{g^{\prime}}{g} \theta \psi_{t}+\lambda \theta \psi_{t}^{2}-\lambda \psi_{t}(c \cdot \nabla \psi) \theta\right) z^{2} \\
\leq & \left\|P_{1} z\right\|^{2}+s^{2} \lambda^{2} \iint \psi_{t}^{2} \theta^{2} z^{2}
\end{aligned}
$$

and hence

$$
s^{2} \lambda^{2} \iint \psi_{t}^{2} \theta^{2} z^{2}-2 s^{2} \lambda \iint \frac{g^{\prime}}{g} \varphi \psi_{t} \theta z^{2}+s \lambda \iint\left(\psi_{t t} \theta+\frac{g^{\prime}}{g} \theta \psi_{t}+\lambda \theta \psi_{t}\left(\psi_{t}-c \cdot \nabla \psi\right)\right) z^{2} \leq\left\|P_{1} z\right\|^{2} .
$$


It follows that

$$
\begin{gathered}
\left\|e^{-s \varphi} P w\right\|^{2}=\left\|P_{1} z\right\|^{2}+\left\|P_{2} z\right\|^{2}+2\left(P_{1} z, P_{2} z\right) \\
\geq s^{2} \lambda^{2} \iint\left(\psi_{t}^{2}+(c \cdot \nabla \psi)^{2}-2 \psi_{t} c \cdot \nabla \psi\right) \theta^{2} z^{2} \\
\quad+2 s^{2} \lambda \iint \frac{g^{\prime}}{g} \varphi \theta\left(-\psi_{t}+c \cdot \nabla \psi\right) z^{2} \\
\quad+s \lambda^{2} \iint\left(-\psi_{t} c \cdot \nabla \psi+(c \cdot \nabla \psi)^{2}+\psi_{t}\left(\psi_{t}-c \cdot \nabla \psi\right)\right) \theta z^{2} \\
\quad+s \lambda \iint\left(-\frac{g^{\prime}}{g} c \cdot \nabla \psi+c \cdot \nabla(c \cdot \nabla \psi)+\psi_{t t}+\frac{g^{\prime}}{g} \psi_{t}\right) \theta z^{2} \\
=s \lambda^{2} \iint\left(\psi_{t}-c \cdot \nabla \psi\right)^{2} \theta^{2} z^{2}+s \lambda^{2} \iint\left(\psi_{t}-c \cdot \nabla \psi\right)^{2} \theta z^{2} \\
\quad+s \lambda \iint\left(\frac{-g^{\prime}}{g}\right)\left(\psi_{t}-c \cdot \nabla \psi\right) \theta(2 s \varphi-1) z^{2} \\
\quad+s \lambda \iint\left(\psi_{t t}+c \cdot \nabla(c \cdot \nabla \psi)\right) \theta z^{2} .
\end{gathered}
$$

The two first integral terms of the r.h.s. being nonnegative, we arrive at

$$
\left\|e^{-s \varphi} P w\right\|^{2} \geq s \lambda \iint\left(\frac{-g^{\prime}}{g}\right)\left(\psi_{t}-c \cdot \nabla \psi\right) \theta(2 s \varphi-1) z^{2}+s \lambda \iint\left(\psi_{t t}+c \cdot \nabla(c \cdot \nabla \psi)\right) \theta z^{2} .
$$

Noticing that

$$
\varphi(x, t)=g(t) e^{\lambda \psi(x, t)}\left(e^{\frac{3}{2} \lambda\|\psi\|_{L^{\infty}}-\lambda \psi(x, t)}-1\right) \geq e^{-K T^{2}} \frac{\lambda\|\psi\|_{L^{\infty}}}{2},
$$

we have that $s \varphi \geq 1$ for $\lambda \geq \lambda_{0}=1$ and $s \geq s_{0}$ with $s_{0}$ large enough. Using (3.7)-(3.8) and the fact that

$$
g^{\prime}(t) \begin{cases}\leq 0 & \text { if } t \in(0, \sigma), \\ =0 & \text { if } t \in[\sigma, T-\sigma], \\ \geq 0 & \text { if } t \in(T-\sigma, T),\end{cases}
$$

we infer that for $\lambda \geq \lambda_{0}$ and $s \geq s_{0}$

$$
s \lambda \iint\left(\frac{-g^{\prime}}{g}\right)\left(\psi_{t}-c \cdot \nabla \psi\right) \theta(2 s \varphi-1) z^{2} \geq s \lambda \int_{0}^{T} \int_{\omega_{0}}\left(\frac{-g^{\prime}}{g}\right)\left(\psi_{t}-c \cdot \nabla \psi\right) \theta(2 s \varphi-1) z^{2},
$$

and hence

$$
s \lambda \iint\left(\psi_{t t}+c \cdot \nabla(c \cdot \nabla \psi)\right) \theta z^{2} \leq\left\|e^{-s \varphi} P w\right\|^{2}+C s^{2} \lambda \int_{0}^{T} \int_{\omega_{0}} \frac{\left|g^{\prime}\right|}{g} \theta \varphi|z|^{2} .
$$

Using (3.9), we obtain for $\lambda \geq \lambda_{0}$ and $s \geq s_{0}$

$$
s \lambda \iint \theta z^{2} \leq C\left(\left\|e^{-s \varphi} P w\right\|^{2}+s \lambda \int_{0}^{T} \int_{\omega_{0}}\left(s \frac{\left|g^{\prime}\right|}{g} \varphi+1\right) \theta z^{2} d x d t\right) .
$$

Replacing $z$ by $e^{-s \varphi} w$ results in (3.10), which is thus established when $w \in H^{1}\left(\mathbb{T}^{2} \times(0, T)\right)$.

We claim that (3.10) is still true when $w$ and $f=-w_{t}+c \cdot \nabla w$ are in $L^{2}\left(0, T, L^{2}\left(\mathbb{T}^{2}\right)\right)$. Indeed, in that case $w \in C\left([0, T], L^{2}\left(\mathbb{T}^{2}\right)\right)$, and if $\left(w_{T}^{n}\right)$ and $\left(f^{n}\right)$ are two sequences in $H^{1}\left(\mathbb{T}^{2}\right)$ and $L^{2}\left(0, T, H^{1}\left(\mathbb{T}^{2}\right)\right)$, 
respectively, such that

$$
\begin{array}{rll}
w_{T}^{n} & \rightarrow w(T) & \text { in } L^{2}\left(\mathbb{T}^{2}\right), \\
f^{n} & \rightarrow f & \text { in } L^{2}\left(0, T, L^{2}\left(\mathbb{T}^{2}\right)\right),
\end{array}
$$

then the solution $w^{n} \in C\left([0, T], H^{1}\left(\mathbb{T}^{2}\right)\right)$ of

$$
\begin{aligned}
-w_{t}^{n}+c \cdot \nabla w^{n} & =f^{n}, \\
w^{n}(T) & =w_{T}^{n}
\end{aligned}
$$

satisfies $w^{n} \in H^{1}\left(\mathbb{T}^{2} \times(0, T)\right)$ and $w^{n} \rightarrow w$ in $C\left([0, T], L^{2}\left(\mathbb{T}^{2}\right)\right)$, so that we can apply (3.10) to $w^{n}$ and pass to the limit $n \rightarrow+\infty$ in (3.10). The proof of Proposition 3.1 is complete.

\subsection{Carleman estimate for the parabolic equation.}

Proposition 3.2. Let $T, \sigma, K, \widetilde{K}, \omega_{0}, \psi_{0}, \psi, g, \varphi$ and $\theta$ be as in Proposition 3.1. Then there exist some constants $\lambda_{1} \geq \lambda_{0}, s_{1} \geq s_{0}$ and $C_{1}>0$ such that for all $\lambda \geq \lambda_{1}$, all $s \geq s_{1}$ and all $v \in C\left([0, T], L^{2}\left(\mathbb{T}^{2}\right)\right)$ with $v_{t}+\Delta v \in L^{2}\left(0, T, L^{2}\left(\mathbb{T}^{2}\right)\right)$, the following holds

$$
\begin{aligned}
\int_{0}^{T} \int_{\mathbb{T}^{2}}\left[(s \theta)^{-1}\left(|\Delta v|^{2}+\left|v_{t}\right|^{2}\right)\right. & \left.+\lambda^{2}(s \theta)|\nabla v|^{2}+\lambda^{4}(s \theta)^{3}|v|^{2}\right] e^{-2 s \varphi} d x d t \\
\leq & C_{1}\left(\int_{0}^{T} \int_{\mathbb{T}^{2}}\left|v_{t}+\Delta v\right|^{2} e^{-2 s \varphi} d x d t+\int_{0}^{T} \int_{\omega_{0}} \lambda^{4}(s \theta)^{3}|v|^{2} e^{-2 s \varphi} d x d t\right) .
\end{aligned}
$$

Proof. We follow closely $[4,15]$. Setting $v_{T}=v(., T) \in L^{2}\left(\mathbb{T}^{2}\right)$, we see that the function $v$ solves the backward parabolic equation

$$
\begin{aligned}
-v_{t}-\Delta v & =f, \\
v(., T) & =v_{T},
\end{aligned}
$$

for some function $f \in L^{2}\left(0, T, L^{2}\left(\mathbb{T}^{2}\right)\right)$. Assume first that $v_{T} \in H^{2}\left(\mathbb{T}^{2}\right)$ and $f \in H^{1}\left(0, T, L^{2}\left(\mathbb{T}^{2}\right)\right)$ so that

$$
v \in L^{\infty}\left(0, T, H^{2}\left(\mathbb{T}^{2}\right)\right) \cap H^{1}\left(0, T, H^{1}\left(\mathbb{T}^{2}\right)\right) .
$$

Let $y=e^{-s \varphi} v$ and $P=\partial_{t}+\Delta$. Then

$$
e^{-s \varphi} P v=e^{-s \varphi} P\left(e^{s \varphi} y\right)=P_{s} y+P_{a} y,
$$

where

$$
\begin{aligned}
P_{s} y & =\Delta y+\left(s \varphi_{t}+s^{2}|\nabla \varphi|^{2}\right) y, \\
P_{a} y & =y_{t}+2 s \nabla \varphi \cdot \nabla y+s(\Delta \varphi) y,
\end{aligned}
$$

denote the (formal) self-adjoint and skew-adjoint parts of $e^{-s \varphi} P\left(e^{s \varphi}\right.$.), respectively. It follows that

$$
\left\|e^{-s \varphi} P v\right\|^{2}=\left\|P_{s} y\right\|^{2}+\left\|P_{a} y\right\|^{2}+2\left(P_{s} y, P_{a} y\right),
$$

where we still write $(f, g)=\int_{0}^{T} \int_{\mathbb{T}^{2}} f g d x d t,\|f\|^{2}=(f, f)$. Again, $\int_{0}^{T} \int_{\mathbb{T}^{2}} f(x, t) d x d t$ will be denoted $\iint f$ for the sake of shortness. We have that

$$
\begin{aligned}
& \left(P_{s} y, P_{a} y\right)=\left(\Delta y, y_{t}\right)+(\Delta y, 2 s \nabla \varphi \cdot \nabla y)+(\Delta y, s(\Delta \varphi) y)+\left(s \varphi_{t} y+s^{2}|\nabla \varphi|^{2} y, y_{t}\right) \\
& \quad+\left(s \varphi_{t} y+s^{2}|\nabla \varphi|^{2} y, 2 s \nabla \varphi \cdot \nabla y\right)+\left(s \varphi_{t} y+s^{2}|\nabla \varphi|^{2} y, s(\Delta \varphi) y\right)=: I_{1}+I_{2}+I_{3}+I_{4}+I_{5}+I_{6} .
\end{aligned}
$$


First, observe that

$$
I_{1}=-\iint \nabla y \cdot \nabla y_{t}=0
$$

Using the convention of repeated indices and denoting $\partial_{i}=\partial / \partial x_{i}$, we obtain that

$$
\begin{aligned}
I_{2} & =2 s \iint \partial_{j}^{2} y \partial_{i} \varphi \partial_{i} y \\
& =-2 s \iint \partial_{j} y\left(\partial_{j} \partial_{i} \varphi \partial_{i} y+\partial_{i} \varphi \partial_{j} \partial_{i} y\right) .
\end{aligned}
$$

It follows that

$$
\begin{aligned}
I_{2} & =-2 s \iint \partial_{j} \partial_{i} \varphi \partial_{j} y \partial_{i} y-s \iint \partial_{i} \varphi \partial_{i}\left(\left|\partial_{j} y\right|^{2}\right) \\
& =-2 s \iint \partial_{j} \partial_{i} \varphi \partial_{j} y \partial_{i} y+s \iint \Delta \varphi|\nabla y|^{2} .
\end{aligned}
$$

On the other hand, doing integrations by parts in $x$ yields

$$
I_{3}=-s \iint \nabla y \cdot(y \nabla(\Delta \varphi)+(\Delta \varphi) \nabla y)=s \iint \Delta^{2} \varphi \frac{|y|^{2}}{2}-s \iint \Delta \varphi|\nabla y|^{2} .
$$

Integrating by parts with respect to $t$ in $I_{4}$ gives

$$
I_{4}=-\iint\left(s \varphi_{t t}+s^{2} \partial_{t}|\nabla \varphi|^{2}\right) \frac{|y|^{2}}{2}
$$

Integrating by parts with respect to $x$ in $I_{5}$ yields

$$
I_{5}=-\iint s^{2} \nabla \cdot\left(\varphi_{t} \nabla \varphi\right)|y|^{2}-\iint s^{3} \nabla \cdot\left(|\nabla \varphi|^{2} \nabla \varphi\right)|y|^{2} .
$$

Gathering (3.18)-(3.22), we infer that

$$
2\left(P_{s} y, P_{a} y\right)=-4 s \iint \partial_{j} \partial_{i} \varphi \partial_{j} y \partial_{i} y+\iint|y|^{2}\left[s\left(\Delta^{2} \varphi-\varphi_{t t}\right)-2 s^{2} \partial_{t}|\nabla \varphi|^{2}-2 s^{3} \nabla \varphi \cdot \nabla|\nabla \varphi|^{2}\right] .
$$

Consequently, (3.17) may be rewritten

$$
\left\|e^{-s \varphi} P v\right\|^{2}=\left\|P_{s} y\right\|^{2}+|| P_{a} y \|^{2}-4 s \iint \partial_{j} \partial_{i} \varphi \partial_{j} v \partial_{i} v+\iint|v|^{2}\left[s\left(\Delta^{2} \varphi-\varphi_{t t}\right)-2 s^{2} \partial_{t}|\nabla \varphi|^{2}-2 s^{3} \nabla \varphi \cdot \nabla|\nabla \varphi|^{2}\right] .
$$

CLAIM 1. There exist some numbers $\lambda_{1} \geq \lambda_{0}, s_{1} \geq s_{0}$ and $A \in(0,1)$ such that for all $\lambda \geq \lambda_{1}$ and all $s \geq s_{1}$,

$$
\begin{aligned}
\iint|y|^{2}\left[s\left(\Delta^{2} \varphi-\varphi_{t t}\right)-2 s^{2} \partial_{t}|\nabla \varphi|^{2}-2 s^{3} \nabla \varphi\right. & \left.\cdot \nabla|\nabla \varphi|^{2}\right] \\
& +A^{-1} \lambda s^{3} \int_{0}^{T} \int_{\omega_{0}}(\lambda \theta)^{3}|y|^{2} \geq A \lambda s^{3} \iint(\lambda \theta)^{3}|y|^{2} .
\end{aligned}
$$

Proof of Claim 1. Easy computations show that

$$
\partial_{i} \varphi=-\lambda g(t) e^{\lambda \psi} \partial_{i} \psi, \quad \partial_{j} \partial_{i} \varphi=-g(t) e^{\lambda \psi}\left(\lambda^{2} \partial_{i} \psi \partial_{j} \psi+\lambda \partial_{j} \partial_{i} \psi\right)
$$

and

$$
-\nabla|\nabla \varphi|^{2} \cdot \nabla \varphi=-2\left(\partial_{j} \partial_{i} \varphi\right) \partial_{i} \varphi \partial_{j} \varphi=2\left(\lambda g e^{\lambda \psi}\right)^{3}\left(\lambda|\nabla \psi|^{4}+\partial_{j} \partial_{i} \psi \partial_{i} \psi \partial_{j} \psi\right)
$$


It follows from (3.6) that for $\lambda$ large enough, say $\lambda \geq \lambda_{1}$, we have that

$$
\begin{aligned}
-\nabla|\nabla \varphi|^{2} \cdot \nabla \varphi & \geq A \lambda(\lambda \theta)^{3}, \quad t \in[0, T], x \in \mathbb{T}^{2} \backslash \omega_{0}, \\
\left.|\nabla| \nabla \varphi\right|^{2} \cdot \nabla \varphi \mid & \leq A^{-1} \lambda(\lambda \theta)^{3}, \quad t \in[0, T], x \in \mathbb{T}^{2} \backslash \omega_{0},
\end{aligned}
$$

for some constant $A \in(0,1)$. It follows from (3.2) that

$$
\left|\Delta^{2} \varphi\right|+\left|\varphi_{t t}\right|+\left.\left|\partial_{t}\right| \nabla \varphi\right|^{2} \mid \leq C \lambda(\lambda \theta)^{3}, \quad t \in[0, T], x \in \bar{\Omega} .
$$

Therefore, we infer that for $s$ large enough, say $s \geq s_{1}$, and for all $\lambda \geq \lambda_{1}$ we have that

$$
\begin{aligned}
s\left(\Delta^{2} \varphi-\varphi_{t t}\right)-2 s^{2} \partial_{t}|\nabla \varphi|^{2}-2 s^{3} \nabla \varphi \cdot \nabla|\nabla \varphi|^{2} & \geq A \lambda s^{3}(\lambda \theta)^{3}, \quad t \in[0, T], x \in \mathbb{T}^{2} \backslash \omega_{0}, \\
\left.\left|s\left(\Delta^{2} \varphi-\varphi_{t t}\right)-2 s^{2} \partial_{t}\right| \nabla \varphi\right|^{2}-2 s^{3} \nabla \varphi \cdot \nabla|\nabla \varphi|^{2} \mid & \leq A^{-1} \lambda s^{3}(\lambda \theta)^{3}, \quad t \in[0, T], x \in \mathbb{T}^{2} \backslash \omega_{0} .
\end{aligned}
$$

This gives (3.23) with a possibly decreased value of $A$.

Thus we conclude that

$$
\begin{aligned}
\left\|P_{s} y\right\|^{2}+\left\|P_{a} y\right\|^{2}+A \lambda s^{3} \iint & (\lambda \theta)^{3}|y|^{2} \\
& \leq\left\|e^{-s \varphi} P p\right\|^{2}+4 s \iint \partial_{j} \partial_{i} \varphi \partial_{j} y \partial_{i} y+A^{-1} \lambda s^{3} \int_{0}^{T} \int_{\omega_{0}}(\lambda \theta)^{3}|y|^{2} .
\end{aligned}
$$

CLAIM 2. There exist some numbers $\lambda_{2} \geq \lambda_{1}$ and $s_{2} \geq s_{1}$ such that for all $\lambda \geq \lambda_{2}$ and all $s \geq s_{2}$,

$$
\lambda s \iint(\lambda \theta)|\nabla y|^{2}+\lambda s^{-1} \iint(\lambda \theta)^{-1}|\Delta y|^{2} \leq C\left(s^{-1}|| P_{s} y||^{2}+\lambda s^{3} \iint(\lambda \theta)^{3}|y|^{2}\right) .
$$

Proof of Claim 2. By (3.15)

$$
\begin{aligned}
s^{-1} \iint(\lambda \theta)^{-1}|\Delta y|^{2} & =\left.\left.s^{-1} \iint(\lambda \theta)^{-1}\left|P_{s} y-s \varphi_{t} y-s^{2}\right| \nabla \varphi\right|^{2} y\right|^{2} \\
& \leq C s^{-1} \iint(\lambda \theta)^{-1}\left(\left|P_{s} y\right|^{2}+s^{2}\left|\varphi_{t}\right|^{2}|y|^{2}+s^{4}(\lambda \theta)^{4}|y|^{2}\right) \\
& \leq C\left(\frac{\left.|| P_{s} y\right|^{2}}{\lambda s}+s^{3} \iint(\lambda \theta)^{3}|y|^{2}\right)
\end{aligned}
$$

provided that $s$ and $\lambda$ are large enough, where we used (3.2) in the last line to bound $\varphi_{t}$. On the other hand,

$$
\begin{aligned}
\lambda s \iint(\lambda \theta)|\nabla y|^{2} & =\lambda s\left\{\iint(\lambda \theta)(-\Delta y) y-\iint(\nabla(\lambda \theta) \cdot \nabla y) y\right\} \\
& \leq \frac{\lambda}{2 s} \iint(\lambda \theta)^{-1}|\Delta y|^{2}+\frac{\lambda s^{3}}{2} \iint(\lambda \theta)^{3}|y|^{2}+\frac{\lambda s}{2} \iint \Delta(\lambda \theta)|y|^{2} \\
& \leq C\left(s^{-1}|| P_{s} y \|^{2}+\lambda s^{3} \iint(\lambda \theta)^{3}|y|^{2}\right),
\end{aligned}
$$

by (3.29), provided that $s \geq s_{2} \geq s_{1}$ and $\lambda \geq \lambda_{2} \geq \lambda_{1}$. Then (3.28) follows from (3.29)-(3.30). 
We infer from (3.27)-(3.28) that

$$
\begin{aligned}
\left\|P_{a} y\right\|^{2}+\lambda s \iint(\lambda \theta)|\nabla y|^{2} & +\lambda s^{-1} \iint(\lambda \theta)^{-1}|\Delta y|^{2}+\lambda s^{3} \iint(\lambda \theta)^{3}|y|^{2} \\
\leq & C\left(\left\|e^{-s \varphi} P p\right\|^{2}+4 s \iint \partial_{j} \partial_{i} \varphi \partial_{j} y \partial_{i} y+A^{-1} \lambda s^{3} \int_{0}^{T} \int_{\omega_{0}}(\lambda \theta)^{3}|y|^{2}\right) .
\end{aligned}
$$

By (3.24),

$$
s \iint \partial_{j} \partial_{i} \varphi \partial_{j} y \partial_{i} y \leq-s \lambda \iint g(t) e^{\lambda \psi} \partial_{j} \partial_{i} \psi \partial_{j} y \partial_{i} y \leq C s \iint(\lambda \theta)|\nabla y|^{2} .
$$

Therefore, for $\lambda$ large enough,

$$
\begin{aligned}
\left\|P_{a} y\right\|^{2}+\lambda s^{3} \iint(\lambda \theta)^{3}|y|^{2}+\lambda s \iint(\lambda \theta)|\nabla y|^{2} & +\lambda s^{-1} \iint(\lambda \theta)^{-1}|\Delta y|^{2} \\
& \leq C\left(\left\|e^{-s \varphi} P p\right\|^{2}+\lambda s^{3} \int_{0}^{T} \int_{\omega_{0}}(\lambda \theta)^{3}|y|^{2}\right) .
\end{aligned}
$$

Using (3.16) and (3.32), we see that for $\lambda$ large enough

$$
\begin{aligned}
\lambda s^{-1} \iint(\lambda \theta)^{-1}\left|y_{t}\right|^{2} & \leq C \lambda s^{-1} \iint(\lambda \theta)^{-1}\left(\left|P_{a} y\right|^{2}+s^{2}|\nabla \varphi|^{2}|\nabla y|^{2}+s^{2}|\Delta \varphi|^{2}|y|^{2}\right) \\
& \leq C\left(\left\|e^{-s \varphi} P p\right\|^{2}+\lambda s^{3} \int_{0}^{T} \int_{\omega_{0}}(\lambda \theta)^{3}|y|^{2}\right) .
\end{aligned}
$$

We conclude that there exists some number $\lambda_{3} \geq \lambda_{2}$ such that for all $\lambda \geq \lambda_{3}$ and all $s \geq s_{2}$, we have

$$
\begin{aligned}
\lambda s^{3} \iint(\lambda \theta)^{3}|y|^{2}+\lambda s \iint(\lambda \theta)|\nabla y|^{2}+\lambda s^{-1} \iint & (\lambda \theta)^{-1}\left(|\Delta y|^{2}+\left|y_{t}\right|^{2}\right) \\
& \leq C\left(\left\|e^{-s \varphi} P p\right\|^{2}+\lambda s^{3} \int_{0}^{T} \int_{\omega_{0}}(\lambda \theta)^{3}|y|^{2}\right) .
\end{aligned}
$$

Replacing $y$ by $e^{-s \varphi} v$ in (3.33) gives at once (3.12). The proof of Proposition 3.2 is complete for $v_{T} \in$ $H^{2}(\mathbb{T})$ and $f=-v_{t}-\Delta v \in H^{1}\left(0, T, L^{2}\left(\mathbb{T}^{2}\right)\right)$. The general case $\left(v_{T} \in L^{2}\left(\mathbb{T}^{2}\right)\right.$ and $f \in L^{2}\left(0, T, L^{2}\left(\mathbb{T}^{2}\right)\right)$ follows by density.

We are now in a position to establish the observability inequality for the adjoint system (1.34)-(1.37).

Proposition 3.3. Let $T, \sigma, K, \widetilde{K}, \omega_{0}, \psi_{0}, \psi, g, \varphi$ and $\theta$ be as in Proposition 3.1. Pick any open set $\omega$ with $\overline{\omega_{0}} \subset \omega$. Then there exist some constants $\lambda_{2} \geq \lambda_{1}, s_{2} \geq s_{1}$ and $C_{2}>0$ such that for all $\lambda \geq \lambda_{2}$, all $s \geq s_{2}$ and all $\left(v_{T}, w_{T}\right) \in\left[L^{2}\left(\mathbb{T}^{2}\right)\right]^{2}$, if $(v, w)$ denotes the solution of (1.34)-(1.37), then we have

$$
\begin{aligned}
\int_{0}^{T} \int_{\mathbb{T}^{2}}\left[\lambda s \theta|w|^{2}+(s \theta)^{-1}\left(|\Delta v|^{2}+\left|v_{t}\right|^{2}\right)+\lambda^{2}(s \theta)|\nabla v|^{2}+\right. & \left.\lambda^{4}(s \theta)^{3}|v|^{2}\right] e^{-2 s \varphi} d x d t \\
& \leq C_{2} \int_{0}^{T} \int_{\omega} \lambda^{8}(s \theta)^{7}|w|^{2} e^{-2 s \varphi} d x d t
\end{aligned}
$$

As a consequence, there is a constant $C_{o b s}>0$ independent of $\left(v_{T}, w_{T}\right)$ such that

$$
\int_{\mathbb{T}^{2}}\left(|v(x, 0)|^{2}+|w(x, 0)|^{2}\right) d x \leq C_{o b s} \int_{0}^{T} \int_{\omega}|w(x, t)|^{2} d x d t .
$$


Proof. It is clear that $v \in C\left([0, T], L^{2}\left(\mathbb{T}^{2}\right)\right) \cap L^{2}\left(0, T, H^{1}\left(\mathbb{T}^{2}\right)\right)$ and that $w \in L^{2}\left(0, T, L^{2}\left(\mathbb{T}^{2}\right)\right)$, so that by (1.34)-(1.35), $v_{t}+\Delta v \in L^{2}\left(0, T, L^{2}\left(\mathbb{T}^{2}\right)\right)$ and $-w_{t}+c \cdot \nabla w \in L^{2}\left(0, T, L^{2}\left(\mathbb{T}^{2}\right)\right)$. It follows then from (3.10) and (3.12) that we have

$$
\begin{aligned}
& \int_{0}^{T} \int_{\mathbb{T}^{2}}\left[\lambda s \theta|w|^{2} e^{-2 s \varphi} d x d t+(s \theta)^{-1}\left(|\Delta v|^{2}+\left|v_{t}\right|^{2}\right)+\lambda^{2}(s \theta)|\nabla v|^{2}+\lambda^{4}(s \theta)^{3}|v|^{2}\right] e^{-2 s \varphi} d x d t \\
& \leq C\left(\int_{0}^{T} \int_{\mathbb{T}^{2}}\left|-w_{t}+c \cdot \nabla w\right|^{2} e^{-2 s \varphi} d x d t+\int_{0}^{T} \int_{\omega_{0}} \lambda s \theta|w|^{2}\left(s \frac{\left|g^{\prime}\right|}{g} \varphi+1\right) e^{-2 s \varphi} d x d t\right. \\
& \left.\quad+\int_{0}^{T} \int_{\mathbb{T}^{2}}\left|v_{t}+\Delta v\right|^{2} e^{-2 s \varphi} d x d t+\int_{0}^{T} \int_{\omega_{0}} \lambda^{4}(s \theta)^{3}|v|^{2} e^{-2 s \varphi} d x d t\right) \\
& \leq C\left(\int_{0}^{T} \int_{\mathbb{T}^{2}}|v-w|^{2} e^{-2 s \varphi} d x d t+\int_{0}^{T} \int_{\omega_{0}} \lambda s \theta|w|^{2}\left(s \frac{\left|g^{\prime}\right|}{g} \varphi+1\right) e^{-2 s \varphi} d x d t\right. \\
& \left.\quad+\int_{0}^{T} \int_{\mathbb{T}^{2}}|c \cdot \nabla v+(b(x+t c)-1)(v-w)|^{2} e^{-2 s \varphi} d x d t+\int_{0}^{T} \int_{\omega_{0}} \lambda^{4}(s \theta)^{3}|v|^{2} e^{-2 s \varphi} d x d t\right),
\end{aligned}
$$

where $C:=\max \left(C_{0}, C_{1}\right)$. In what follows, $C$ denotes a constant (which does not depend on $s, \lambda, v_{T}, w_{T}$ ) that may vary from line to line. Taking $s \geq s_{2} \geq s_{1}$ and $\lambda \geq \lambda_{2} \geq \lambda_{1}$ with $s_{2}, \lambda_{2}$ large enough, we can absorb the first and third integral terms in the right hand side of the last equation, so that

$$
\begin{aligned}
& \int_{0}^{T} \int_{\mathbb{T}^{2}}\left[\lambda s \theta|w|^{2} e^{-2 s \varphi}+(s \theta)^{-1}\left(|\Delta v|^{2}+\left|v_{t}\right|^{2}\right)+\lambda^{2}(s \theta)|\nabla v|^{2}+\lambda^{4}(s \theta)^{3}|v|^{2}\right] e^{-2 s \varphi} d x d t \\
& \leq C\left(\int_{0}^{T} \int_{\omega_{0}} \lambda s \theta|w|^{2}\left(s \frac{\left|g^{\prime}\right|}{g} \varphi+1\right) e^{-2 s \varphi} d x d t+\int_{0}^{T} \int_{\omega_{0}} \lambda^{4}(s \theta)^{3}|v|^{2} e^{-2 s \varphi} d x d t\right) .
\end{aligned}
$$

It remains to eliminate the last integral term. To do this, following [17], we derive local energy estimates. Introduce a cut-off function $\chi$ such that

$$
\begin{array}{rr}
\chi \in C_{0}^{\infty}(\omega), & \\
0 \leq \chi(x) \leq 1, & x \in \mathbb{T}^{2}, \\
\chi(x)=1, & x \in \omega_{0} .
\end{array}
$$

We have that

$$
\int_{0}^{T} \int_{\omega_{0}} \lambda^{4}(s \theta)^{3}|v|^{2} e^{-2 s \varphi} d x d t \leq \int_{0}^{T} \int_{\mathbb{T}^{2}} \chi \lambda^{4}(s \theta)^{3}|v|^{2} e^{-2 s \varphi} d x d t
$$

Using (1.35), we obtain that

$$
\begin{aligned}
\int_{0}^{T} \int_{\mathbb{T}^{2}} \chi \lambda^{4}(s \theta)^{3}|v|^{2} e^{-2 s \varphi} d x d t= & \int_{0}^{T} \int_{\mathbb{T}^{2}} \chi \lambda^{4}(s \theta)^{3} v w e^{-2 s \varphi} d x d t \\
& +\int_{0}^{T} \int_{\mathbb{T}^{2}} \chi \lambda^{4}(s \theta)^{3} v\left(-w_{t}\right) e^{-2 s \varphi} d x d t \\
& +\int_{0}^{T} \int_{\mathbb{T}^{2}} \chi \lambda^{4}(s \theta)^{3} v(c \cdot \nabla w) e^{-2 s \varphi} d x d t \\
=: & M_{1}+M_{2}+M_{3} .
\end{aligned}
$$


Using Cauchy-Schwarz inequality and (3.37)-(3.38), we have that for every $\varepsilon>0$

$$
\left|M_{1}\right| \leq \varepsilon \int_{0}^{T} \int_{\mathbb{T}^{2}} \lambda^{4}(s \theta)^{3}|v|^{2} e^{-2 s \varphi} d x d t+\frac{1}{4 \varepsilon} \int_{0}^{T} \int_{\omega} \lambda^{4}(s \theta)^{3}|w|^{2} e^{-2 s \varphi} d x d t .
$$

On the other hand, integrating by parts with respect to $t$ in $M_{2}$ yields

$$
\begin{aligned}
M_{2} & =\int_{0}^{T} \int_{\mathbb{T}^{2}} \chi \lambda^{4}(s \theta)^{3} v_{t} w e^{-2 s \varphi} d x d t+\int_{0}^{T} \int_{\mathbb{T}^{2}} \chi \lambda^{4}\left(3 s^{3} \theta^{2} \theta_{t}-2 s^{4} \varphi_{t} \theta^{3}\right) v w e^{-2 s \varphi} d x d t \\
& =M_{2}^{1}+M_{2}^{2} .
\end{aligned}
$$

For $M_{2}^{1}$, we notice that for every $\varepsilon>0$ we have that

$$
\left|M_{2}^{1}\right| \leq \varepsilon \int_{0}^{T} \int_{\mathbb{T}^{2}}(s \theta)^{-1}\left|v_{t}\right|^{2} e^{-2 s \varphi} d x d t+\frac{1}{4 \varepsilon} \int_{0}^{T} \int_{\omega} \lambda^{8}(s \theta)^{7}|w|^{2} e^{-2 s \varphi} d x d t .
$$

Since $\left|\theta_{t}\right|+\left|\varphi_{t}\right| \leq C \lambda \theta^{2}$, we infer that for every $\varepsilon>0$

$$
\begin{aligned}
\left|M_{2}^{2}\right| & \leq C \int_{0}^{T} \int_{\mathbb{T}^{2}} \chi s^{4}(\lambda \theta)^{5}|v w| e^{-2 s \varphi} d x d t \\
& \leq \varepsilon \int_{0}^{T} \int_{\mathbb{T}^{2}} \lambda^{4}(s \theta)^{3}|v|^{2} e^{-2 s \varphi} d x d t+\frac{C}{\varepsilon s^{2}} \int_{0}^{T} \int_{\omega} \lambda^{6}(s \theta)^{7}|w|^{2} e^{-2 s \varphi} d x d t .
\end{aligned}
$$

Similarly, integrating by parts with respect to $x$ in $M_{3}$ yields

$$
\begin{aligned}
M_{3}= & -\int_{0}^{T} \int_{\mathbb{T}^{2}}(c \cdot \nabla \chi) \lambda^{4}(s \theta)^{3} v w e^{-2 s \varphi} d x d t-\int_{0}^{T} \int_{\mathbb{T}^{2}} \chi \lambda^{4}(s \theta)^{3}(c \cdot \nabla v) w e^{-2 s \varphi} d x d t \\
& \quad+\int_{0}^{T} \int_{\mathbb{T}^{2}} \chi \lambda^{4}\left(3 s^{3} \theta^{2}(c \cdot \nabla \theta)-2 s^{4} \theta^{3}(c \cdot \nabla \varphi)\right) v w e^{-2 s \varphi} d x d t \\
= & -M_{3}^{1}-M_{3}^{2}+M_{3}^{2} .
\end{aligned}
$$

For any $\varepsilon>0$, we have that

$$
\left|M_{3}^{1}\right| \leq \varepsilon \int_{0}^{T} \int_{\mathbb{T}^{2}} \lambda^{4}(s \theta)^{3}|v|^{2} e^{-2 s \varphi} d x d t+\frac{C}{\varepsilon} \int_{0}^{T} \int_{\omega} \lambda^{4}(s \theta)^{3}|w|^{2} e^{-2 s \varphi} d x d t
$$

and

$$
\left|M_{3}^{2}\right| \leq \varepsilon \int_{0}^{T} \int_{\mathbb{T}^{2}} \lambda^{2}(s \theta)|\nabla v|^{2} e^{-2 s \varphi} d x d t+\frac{C}{\varepsilon} \int_{0}^{T} \int_{\omega} \lambda^{6}(s \theta)^{5}|w|^{2} e^{-2 s \varphi} d x d t .
$$

Finally, since $|\nabla \theta|+|\nabla \varphi| \leq C \lambda \theta$, we have that

$$
\begin{aligned}
\left|M_{3}^{3}\right| & \leq C \int_{0}^{T} \int_{\mathbb{T}^{2}} \chi \lambda^{5}(s \theta)^{4}|v w| e^{-2 s \varphi} d x d t \\
& \leq \varepsilon \int_{0}^{T} \int_{\mathbb{T}^{2}} \lambda^{4}(s \theta)^{3}|v|^{2} e^{-2 s \varphi} d x d t+\frac{C}{\varepsilon} \int_{0}^{T} \int_{\omega} \lambda^{6}(s \theta)^{5}|w|^{2} e^{-2 s \varphi} d x d t .
\end{aligned}
$$

Gathering together (3.36)-(3.47), taking $\varepsilon$ small enough, and noticing that $\left|g^{\prime}\right| \varphi / g \leq C \theta^{2}$, we obtain (3.34). It remains to prove the observability inequality (3.35). Pick any $\left(v_{t}, w_{T}\right) \in\left[L^{2}\left(\mathbb{T}^{2}\right)\right]^{2}$, and denote by $(v, w)$ the solution of (1.34)-(1.37). Note that $v \in C\left([0, T], L^{2}\left(\mathbb{T}^{2}\right)\right) \cap L^{2}\left(0, T, H^{1}\left(\mathbb{T}^{2}\right)\right)$ and that $w \in$ $C\left([0, T], L^{2}\left(\mathbb{T}^{2}\right)\right)$. Using classical semigroup estimates, one derives at once (3.35) from (3.34). 


\section{Proofs of Theorems 1.1, 1.2 AND 1.3}

4.1. Proof of Theorem 1.1. Pick some open sets $\omega_{1}$ and $\omega$ as in Theorem 1.1, and pick $T>1-$ $\min _{[0,1]} \rho_{2}+\max _{[0,1]} \rho_{1}$. Let $\hat{\delta}>0$ and $\sigma>0$ be given (small) numbers, to be chosen later on. Set $K:=1-\hat{\delta}$ and $\tau:=1+K \sigma-\min _{[0,1]} \rho_{2}$. Pick a function $\psi_{0}$ as given in Proposition 2.1. Let $\psi$ be as in (3.1) with $t_{0}:=T$ and $\tilde{K}$ large enough so that (3.2) holds. Then for $x \in \mathbb{T}^{2} \backslash \omega_{0}$, we have that

$$
\begin{array}{cc}
-2 K(t-T)-c \cdot \nabla \psi_{0}(x) \geq 2 K(T-\sigma)-2\left(\tau+\max _{x_{2} \in[0,1]} \rho_{1}\left(x_{2}\right)\right), & \text { for } t \in(0, \sigma), \\
-2 K(t-T)-c \cdot \nabla \psi_{0}(x) \leq 2 K \sigma-2\left(\tau-1+\min _{x_{2} \in[0,1]} \rho_{2}\left(x_{2}\right)\right), & \text { for } t \in(T-\sigma, T), \\
c \cdot \nabla\left(c \cdot \nabla \psi_{0}\right)(x)-2 K=2-2(1-\hat{\delta})>\hat{\delta} . &
\end{array}
$$

Then the conditions (3.8)-(3.9) are satisfied, and (3.7) holds provided that

$$
T \geq 2 \sigma+K^{-1}\left(1-\min _{x_{2} \in[0,1]} \rho_{2}\left(x_{2}\right)+\max _{x_{2} \in[0,1]} \rho_{1}\left(x_{2}\right)\right),
$$

a condition which is fulfilled for $\hat{\delta}$ and $\sigma$ small enough. Thus we can apply Proposition 3.1, Proposition 3.2 and Proposition 3.3. The observability inequality (3.35) gives the desired null controllability of system (1.30)-(1.33).

4.2. Proof of Theorem 1.2. Let $c=(1, p / q), \omega_{0}$ and $\omega$ be as in Theorem 1.2. Let $\psi_{0}$ be the function given by Proposition 2.3. Pick any $T>q$. Then we can find some numbers $\tau \in(0,1], \sigma \in$ $(0, \min (1, T / 2))$ and $\hat{\delta} \in(0,1)$ such that, taking $K:=1-\hat{\delta}$, we have

$$
\begin{array}{r}
T \geq \sigma+\frac{q+\tau}{K}, \\
\tau-K \sigma \geq 0 .
\end{array}
$$

Let $\psi$ be as in (3.1) with $t_{0}:=T$ and $\tilde{K}$ large enough so that (3.2) holds. Then for $x \in \mathbb{T}^{2} \backslash \omega_{0}$, we have that

$$
\begin{aligned}
-2 K(t-T)-c \cdot \nabla \psi_{0}(x) \geq 2 K(T-\sigma)-(2 q+2 \tau) \geq 0, & \text { for } t \in(0, \sigma), \\
-2 K(t-T)-c \cdot \nabla \psi_{0}(x) \leq 2 K \sigma-2 \tau \leq 0, & \text { for } t \in(T-\sigma, T), \\
c \cdot \nabla\left(c \cdot \nabla \psi_{0}\right)(x)-2 K=2-2(1-\hat{\delta})>\hat{\delta} . &
\end{aligned}
$$

the conditions (3.7), (3.8) and (3.9) are satisfied. Thus we can apply Proposition 3.1, Proposition 3.2 and Proposition 3.3. The observability inequality (3.35) gives the desired null controllability of system (1.30)-(1.33).

4.3. Proof of Theorem 1.3. Let $c=(1, \zeta)$ with $\zeta \in \mathcal{J}_{\delta}$ and $f_{2}(\delta, \zeta)<1$. Pick any nonempty open set $\omega$ in $\mathbb{T}^{2}$. We can assume that $0 \in \omega$ without loss of generality. Pick any open set $\omega_{0}$ with $0 \in \omega_{0}$ and $\overline{\omega_{0}} \subset \omega$. Let the pair $(p, q)$ and the function $\psi_{0}$ be as given by Proposition 2.4. Note that

$$
\left|c \cdot \nabla\left(c \cdot \nabla \psi_{0}\right)(x)-2\right| \leq f_{2}(\delta, \zeta)<1, \quad x \in \mathbb{T}^{2} \backslash \omega_{0},
$$

and hence there is some number $\hat{\delta} \in(0,1)$ such that

$$
c \cdot \nabla\left(c \cdot \nabla \psi_{0}\right)(x)>2-\hat{\delta}, \quad x \in \mathbb{T}^{2} \backslash \omega_{0} .
$$


Pick any $\tau \in(0,1], \sigma \in(0, \min (1, T / 2))$ and $K:=1-\hat{\delta}$. Let $\psi$ be as in (3.1) with $t_{0}:=T / 2$ and $\tilde{K}$ large enough so that (3.2) holds. Then for $x \in \mathbb{T}^{2} \backslash \omega_{0}$, we have that

$$
\begin{array}{cc}
-2 K\left(t-\frac{T}{2}\right)-c \cdot \nabla \psi_{0}(x) \geq 2 K\left(\frac{T}{2}-\sigma\right)-(2 q+2 \tau), & \text { for } t \in(0, \sigma), \\
-2 K\left(t-\frac{T}{2}\right)-c \cdot \nabla \psi_{0}(x) \leq-2 K\left(\frac{T}{2}-\sigma\right)-2 \tau, & \text { for } t \in(T-\sigma, T), \\
c \cdot \nabla\left(c \cdot \nabla \psi_{0}\right)(x)-2 K>2-\hat{\delta}-2(1-\hat{\delta})=\hat{\delta} . &
\end{array}
$$

It is clear that the conditions (3.7), (3.8) and (3.9) are satisfied for $T>0$ large enough. Thus we can apply Proposition 3.1, Proposition 3.2 and Proposition 3.3. The observability inequality (3.35) gives the desired null controllability of system (1.30)-(1.33).

\section{ACKNOWLEDGEMENTS}

Patricio Guzmán has been partially supported by Basal Project FB0008 and FONDECYT 3180363. Lionel Rosier was partially supported by the ANR project Finite4SoS (ANR-15-CE23-0007).

\section{REFERENCES}

[1] P. Albano, D. Tataru, Carleman estimates and boundary observability for a coupled parabolic-hyperbolic system, Electron. J. Differ. Equ. (2000), No. 22, pp. 1-15.

[2] L. Auslander, R. E. MacKenzie, Introduction to differentiable manifolds. Reprint of the 1977 edition. Dover Publications, Inc., Mineola, NY, 2009.

[3] E. Cerpa, E. Crépeau, On the controllability of the improved Boussinesq equation, SIAM J. Control Optim. 56 (2018), no. 4, 3035-3049.

[4] F. Chaves-Silva, L. Rosier, E. Zuazua, Null controllability of a system of viscoelasticity with a moving control, J. Math. Pures Appl. 101 (2) (2014), pp. 198-222.

[5] F. Chaves-Silva, X. Zhang, E. Zuazua, Controllability of evolution equations with memory, SIAM J. Control Optim. 55 (2017), no. 4, 2437-2459.

[6] A. Fursikov, O. Imanuvilov, Controllability of Evolution Equations, in: Lecture Notes Series, Vol. 34, Seoul National University, Korea, 1996.

[7] P. Gao, Null controllability of the viscous Camassa-Holm equation with moving control, Proc. Indian Acad. Sci. Math. Sci. 126 (1) (2016), pp. 99-108.

[8] M. Golubitsky, V. Guillemin. Stable mappings and their singularities. Graduate Texts in Mathematics, Vol. 14. SpringerVerlag, New York-Heidelberg, 1973.

[9] G. H. Hardy, E. M. Wright. Fifth edition. The Clarendon Press, Oxford University Press, New York, 1979.

[10] S. Lang. Introduction to Diophantine approximations. Second edition. Springer-Verlag, New York, 1995.

[11] P. Martin, L. Rosier, P. Rouchon, Null controllability of the structurally damped wave equation with moving control, SIAM J. Control Optim. 51 (1) (2013), pp. 660-684.

[12] S. Micu, On the controllability of the linearized Benjamin-Bona-Mahony equation, SIAM J. Control Optim. 39 (6) (2001) pp. 1766-1696.

[13] A. Pazy. Semigroups of linear operators and applications to partial differential equations. Applied Mathematical Sciences, 44. Springer-Verlag, New York, 1983.

[14] L. Rosier, P. Rouchon, On the controllability of a wave equation with structural damping, Int. J. Tomogr. Stat. 5 (W07) (2007), pp. 79-84.

[15] L. Rosier, B.-Y. Zhang, Null controllability of the complex Ginzburg-Landeau equation, Ann. Inst. Henri Poincaré, Anal. Non Linéaire 26 (2009) 649-673.

[16] L. Rosier, B.-Y. Zhang, Unique continuation property and control for the Benjamin-Bona-Mahony equation on a periodic domain, J. Differential Equations 254 (1) (2013), pp. 141-178. 
[17] L. de Teresa, Insensitizing controls for a semilinear heat equation, Comm. Partial Differential Equations 25 (2000), no. $1-2,39-72$.

Departamento de Matemática, Universidad Técnica Federico Santa María, Avenida España 1680, Casilla 110-V, Valparaíso, Chile

E-mail address: patricio.guzmanm@usm.cl

Centre Automatique et Systèmes (CAS) and Centre de Robotique, Mines Paristech, PSL Research University, 60 Boulevard Saint-Michel, 75272 Paris Cedex 06, France

E-mail address: lionel.rosier@mines-paristech.fr 